\title{
THE ROLE OF TURBULENCE AND MAGNETIC FIELDS IN SIMULATED FILAMENTARY STRUCTURE
}

\author{
Helen Kirk ${ }^{1,2,3,5}$, Mikhail Klassen ${ }^{2}$, Ralph Pudritz $^{1,2}$, and Samantha Pillsworth ${ }^{2,4}$ \\ ${ }^{1}$ Origins Institute, McMaster University, Hamilton, ON, L8S 4M1, Canada; helen.kirk@nrc-cnrc.gc.ca \\ 2 Department of Physics and Astronomy, McMaster University,Hamilton, ON, L8S 4M1, Canada \\ ${ }^{3}$ National Research Council Canada, Herzberg Astronomy and Astrophysics, Victoria, BC, V9E 2E7, Canada; \\ ${ }^{4}$ Saint Mary's University, Department of Astronomy and Physics, Halifax, NS, B3H 3C3, Canada \\ Received 2014 September 2; accepted 2015 January 20; published 2015 March 24
}

\begin{abstract}
We use numerical simulations of turbulent cluster-forming regions to study the nature of dense filamentary structures in star formation. Using four hydrodynamic and magnetohydrodynamic simulations chosen to match observations, we identify filaments in the resulting column density maps and analyze their properties. We calculate the radial column density profiles of the filaments every $0.05 \mathrm{Myr}$ and fit the profiles with the modified isothermal and pressure-confined isothermal cylinder models, finding reasonable fits for either model. The filaments formed in the simulations have similar radial column density profiles to those observed. Magnetic fields provide additional pressure support to the filaments, making "puffier" filaments less prone to fragmentation than in the pure hydrodynamic case, which continue to condense at a slower rate. In the higher density simulations, the filaments grow faster through the increased importance of gravity. Not all of the filaments identified in the simulations will evolve to form stars: some expand and disperse. Given these different filament evolutionary paths, the trends in bulk filament width as a function of time, magnetic field strength, or density are weak, and all cases are reasonably consistent with the finding of a constant filament width in different star-forming regions. In the simulations, the mean FWHM lies between 0.06 and $0.26 \mathrm{pc}$ for all times and initial conditions, with most lying between 0.1 to $0.15 \mathrm{pc}$; the range in FWHMs is however, larger than seen in typical Herschel analyses. Finally, the filaments display a wealth of substructure similar to the recent discovery of filament bundles in Taurus.
\end{abstract}

Key words: ISM: magnetic fields - methods: numerical - stars: formation - submillimeter: ISM

\section{INTRODUCTION}

Filaments appear to be an important ingredient in the formation of stars. While filaments have been known to be associated with star-forming regions for decades (e.g., Schneider \& Elmegreen 1979; Bally et al. 1987), observations from the Herschel Space Telescope, particularly the Gould Belt (André et al. 2010) and HOBYS (Motte et al. 2010) Legacy Surveys have underlined the prevalence of filamentary structures within star-forming regions. With Herschel's unprecedented ability to sensitively map large areas of the sky, several common properties of filaments have now been identified. First, filaments appear to not be well represented by the Ostriker (1964) equilibrium model of an isothermal cylinder; the column density profile is shallower (e.g., Arzoumanian et al. 2011). This may indicate that magnetic fields (e.g., Fiege \& Pudritz 2000) contribute to supporting the filament from collapse, although Smith et al. (2014) demonstrate that filaments formed in purely turbulent environments also have a similarly shallow slope. Rotation may also lead to a shallower slope (Recchi et al. 2014). Second, the mass per unit length of filaments appears to correlate with star formation activity: filaments with mass per unit length less than the value needed for collapse of an isothermal cylinder (Ostriker 1964) tend to be associated with regions which are forming few if any stars, while filaments with supercritical mass per unit length values tend to be associated with active star-forming regions (e.g., Arzoumanian et al. 2011; Hennemann et al. 2012). What is still unclear, however, is what forces dominate the formation and evolution of the filaments, and how the filaments contribute to star formation. For example, are the filaments formed

\footnotetext{
${ }^{5}$ Banting Fellow.
}

primarily through turbulent shocks, or under the influence of magnetic fields or gravity? Does turbulence control the ability of filaments to fragment into star-forming cores? What forces set the observed (column) density profiles? And do filaments primarily provide a denser collection of gas to promote local star formation (e.g., Hacar \& Tafalla 2011), or do they play a significant role in providing a conduit of mass for the formation of larger stellar clusters, which appear to form preferentially at the intersection of several filaments (see e.g., Myers 2009, 2011; Hennemann et al. 2012; Schneider et al. 2012; Kirk et al. 2013)?

In this paper, we investigate the first of these issues, namely the formation and evolution of filaments, through the analysis of our numerical simulations. We compare the column density properties of filaments formed within four different simulations: higher and lower density, and with and without magnetic fields. These analyses provide a complementary look at simulations to those recently published in Smith et al. (2014), where the influence of different types of turbulence on filament properties was examined, but the effect of the inclusion of magnetic fields or differing initial mean densities was not.

We find that while the largest-scale structures in the gas are set by turbulent motions and appear similar in all four simulations, magnetic fields and gravity do influence the properties of individual filaments. In particular, magnetic fields cushion the initial turbulent gas compressions, leading to filaments which are initially less condensed, and subsequently evolve more slowly (due to the weaker gravitational pull) than the corresponding hydrodynamic case. We note that the simulations we analyze were only able to be run for a few tenths of the global free-fall time, limiting our sensitivity to later-time evolutionary trends. The simulated filaments have properties that are consistent with those measured in real filaments characterized by Herschel, suggesting that the general insights gained with 
these simulations are applicable to real molecular clouds. Finally, turbulence and magnetic fields, and not just the thermal properties of molecular gas, appear to set the critical conditions for gravitational instability leading to star formation.

In what follows, we first discuss our numerical methods and simulations (Section 2), discuss the basic filament properties resulting from the simulations (Section 3), compare various models of filament structure (Section 4), and examine the effects of spatial resolution in characterizing filaments (Section $5)$. We discuss our results and their implications, as well as the limitations of our present analysis in Section 6.

\section{NUMERICAL METHODS}

\subsection{Simulation Setup}

We used the FLASH hydrodynamics code (Fryxell et al. 2000) version 2.5 to perform numerical simulations of molecular clumps, i.e., parsec-scale condensations of gas capable of forming a cluster of stars. FLASH solves the fluid-dynamical equations on an adaptive Eulerian grid, making use of the PARAMESH library (Olson et al. 1999; MacNeice et al. 2000). It includes self-gravity, Lagrangian sink particles to represent gravitationally collapsing cores and (proto)stars (Banerjee et al. 2009; Federrath et al. 2010), and gas cooling by dust and by molecular lines (Banerjee et al. 2006). Stellar properties are self-consistently evolved via a one-zone model (Offner et al. 2009; Klassen et al. 2012).

We initialize our simulation volume with a turbulent velocity field. The turbulence is a mixture of compressive and solenoidal turbulence (Federrath et al. 2008; Girichidis et al. 2011) with a Burgers spectrum, i.e., $E_{k} \propto k^{-2}$ as in Girichidis et al. (2011), and largest modes having a size scale roughly equal to the side length of the simulation box. See also Larson (1981), Boldyrev (2002), and Heyer \& Brunt (2004). The turbulent velocity field has a root-mean-square Mach number of 6 .

We perform a grid of simulations in a cube-shaped volume containing either approximately $500 M_{\odot}$ or $2000 M_{\odot}$ of molecular gas with a power-law density profile scaling as $\rho(r)=\rho_{c} r^{-3 / 2}$. The choice of density profile is motivated by observations of dense gas associated with high-mass star formation (Pirogov 2009); Kauffmann et al. (2010) similarly analyze a suite of dust emission and extinction maps of molecular clouds within the solar neighborhood, and find that those that are not forming high-mass stars obey $\rho(r) \propto r^{-1.63}$. The simulation volume has a side length of $2 \mathrm{pc}$, and the molecular gas is at an initial temperature of $10 \mathrm{~K}$.

These initial conditions were chosen to be representative of nearby molecular clumps, with a focus on NGC 1333, a clusterforming region within the Perseus molecular cloud, located roughly $250 \mathrm{pc}$ away, and currently forming a young cluster of low- and intermediate-mass stars (Walawender et al. 2008). Using a large-scale column density map derived from 2MASSbased extinction, Kirk et al. (2006) estimate that NGC 1333 contains $\sim 1000 M_{\odot}$ within a radius of $\sim 1 \mathrm{pc}$; the simulations contain 500 and $2000 M_{\odot}$ within a 2 pc cube, thus bracketing NGC 1333's mean density. The free-fall time for these simulations is $\sim 1$ and $0.5 \mathrm{Myr}$, respectively. A Mach number of 6 is consistent with the typical ${ }^{13} \mathrm{CO}$ velocity dispersion measured across NGC 1333 reported in Kirk et al. (2010), and we note is also consistent with the standard linewidth-size relationship (Larson 1981). Molecular clumps tend to have temperatures of 10-20 K (Bergin \& Tafalla 2007), and pointed observations toward dense cores in Perseus (Rosolowsky et al. 2008) have a mean temperature of $11 \mathrm{~K}$, although those found in NGC 1333 and other clustered environments tend to have slightly higher values (Schnee et al. 2009; Foster et al. 2009). Similarly, the dust temperature is estimated to be slightly elevated in areas near luminous young protostars (Hatchell et al. 2013). None of these heating effects, however, would have been present prior to the onset of star formation in the region, suggesting that an initial temperature of $10 \mathrm{~K}$ is reasonable.

We used the same initial turbulent velocity field for each simulation, but compared magnetohydrodynamic runs with pure hydro simulations where the magnetic field strength was set to zero. When including magnetic effects, we initialize a magnetic field parallel to the $z$-axis with uniform field strength. We select a field strength for our MHD simulation so our massto-flux ratio is $\lambda \sim 1-2$; this is slightly stronger than the typical range estimated by Crutcher et al. (2010) of $2-3$. The mass-to-flux ratio is given by

$$
\lambda=\frac{M_{\mathrm{tot}}}{\pi R^{2}\langle B\rangle} \frac{\sqrt{G}}{0.13}
$$

where $M_{\text {tot }}$ is the total cloud mass, $R$ the cloud radius, and $\langle B\rangle$ the initial mean magnetic field strength. The factor of 0.13 is required to normalize the flux ratio relative to the critical value where the magnetic field just prevents gravitational collapse (Mouschovias \& Spitzer 1976; Seifried et al. 2011). High-mass star-forming cores typically have values $\lambda \lesssim 5$ (Falgarone et al. 2008; Girart et al. 2009; Beuther et al. 2010).

Table 1 lists the parameters for the grid of simulations run. We note that while stars (sink cells) do form in all of our simulations, as we would expect in reality, the resolution (50 $\mathrm{AU})$ is insufficient to correctly predict the masses of the stars that form; tests we ran with an increased resolution led to a larger number of lower mass stars. This is not a problem for our analysis, as the resolution is more than sufficient to characterize the structure of the filamentary gas at observable scales. Furthermore, the simulations are stopped at an early enough time that stellar feedback would not have had time to influence the evolution of the gas.

\subsection{Filament Identification}

The initial turbulent velocity field quickly results in a highly filamentary structure, as illustrated in Figure 1. We run each of our simulations until the filamentary structure is well-developed; the simulation is stopped at 0.2 to 0.3 free-fall times for the $500 M_{\odot}$ simulations (for the MHD and HD simulations respectively), and 0.13 free-fall times for the $2000 M_{\odot}$ simulations. As FLASH is an adaptive mesh refinement (AMR) code, we first take the output files and map them to a uniform grid, downsampling somewhat to allow the entire grid to fit into memory. Even with the downsampling, our resolution is $\sim 0.002 \mathrm{pc}$, much better than achievable with Herschel for nearby star-forming regions. We then project the density along each of the coordinate axes to create column density maps.

Figure 1 shows the column density in the $\mathrm{X}$ projection for both the $500 M_{\odot}$ and $2000 M_{\odot}$ simulations at all time steps analyzed. Note that the MHD simulation was run for $0.15 \mathrm{Myr}$, while the HD simulation was run for $0.2 \mathrm{Myr}$ for the $500 M_{\odot}$ simulations, giving one additional time step for our HD analyses. In this figure, all the panels have the same dynamic range shown for the grayscale column density, highlighting that 
Table 1

Simulation Parameters

\begin{tabular}{|c|c|c|c|c|c|c|}
\hline \multicolumn{7}{|c|}{ Physical Simulation Parameters } \\
\hline Parameter & & & $500 \mathrm{HYD}$ & $500 \mathrm{MHD}$ & 2000 HYD & $200 \mathrm{OMHD}$ \\
\hline Cloud radius & $(\mathrm{pc})$ & $R_{0}$ & 0.99978 & 0.99978 & 0.99978 & 0.99978 \\
\hline Total cloud mass & $\left(M_{\odot}\right)$ & $M_{\mathrm{tot}}$ & 502.603 & 502.603 & 2152.11 & 2152.11 \\
\hline Mean mass density & $\left(\mathrm{g} \mathrm{cm}^{-3}\right)$ & $\langle\rho\rangle$ & $4.256 \times 10^{-21}$ & $4.256 \times 10^{-21}$ & $1.822 \times 10^{-20}$ & $1.822 \times 10^{-20}$ \\
\hline Mean number density & $\left(\mathrm{cm}^{-3}\right)$ & $\langle n\rangle$ & 1188.98 & 1188.98 & 5091.14 & 5091.14 \\
\hline Mean molecular weight & & $\mu$ & 2.14 & 2.14 & 2.14 & 2.14 \\
\hline Temperature & $(\mathrm{K})$ & $T$ & 10 & 10 & 10 & 10 \\
\hline Sound speed & $\left(\mathrm{km} \mathrm{s}^{-1}\right)$ & $c_{\mathrm{s}}$ & 0.196 & 0.196 & 0.196 & 0.196 \\
\hline rms Mach number & & $\mathcal{M}$ & 6.01 & 6.01 & 6.01 & 6.01 \\
\hline rms turbulent Alfvénic Mach Number & & $\mathcal{M}_{A}$ & 2.1 & 2.1 & 2.2 & 2.2 \\
\hline Mean freefall time & (Myr) & $t_{\mathrm{ff}}$ & 0.74 & 0.74 & 0.370 & 0.370 \\
\hline Sound crossing time & (Myr) & $t_{\mathrm{sc}}$ & 9.96 & 9.96 & 9.96 & 9.96 \\
\hline Turbulent crossing time & (Myr) & $t_{\mathrm{tc}}$ & 1.66 & 1.66 & 1.66 & 1.66 \\
\hline Jeans length & (pc) & $\lambda_{J}$ & 0.413 & 0.413 & 0.199 & 0.199 \\
\hline Jeans volume & $\left(\mathrm{pc}^{3}\right)$ & $V_{\mathrm{J}}$ & 0.294 & 0.294 & 0.033 & 0.033 \\
\hline Jeans mass & $\left(M_{\odot}\right)$ & $M_{J}$ & 4.42 & 4.42 & 2.13 & 2.13 \\
\hline Magnetic field & $(\mu \mathrm{G})$ & $B$ & 0 & 56.7 & 0 & 120.5 \\
\hline Mass-to-flux ratio & & $\lambda$ & $\infty$ & 1.17144 & $\infty$ & 2.35979 \\
\hline Rigid rotation angular frequency & $\left(\operatorname{rad~s}^{-1}\right)$ & $\Omega_{\mathrm{rot}}$ & $1.114 \mathrm{e}-14$ & $1.114 \mathrm{e}-14$ & $1.114 \mathrm{e}-14$ & $1.114 \mathrm{e}-14$ \\
\hline Rotational energy fraction & & $\beta_{\mathrm{rot}}$ & $1.8 \%$ & $1.8 \%$ & $0.4 \%$ & $0.4 \%$ \\
\hline \multicolumn{7}{|c|}{ Numerical Simulation Parameters } \\
\hline Simulation box size & $(\mathrm{pc})$ & $L_{\text {box }}$ & 1.99956 & 1.99956 & 1.99956 & 1.99956 \\
\hline Simulation box volume & $\left(\mathrm{pc}^{3}\right)$ & $V_{\text {box }}$ & 7.99471 & 7.99471 & 7.99471 & 7.99471 \\
\hline Smallest cell size & $(\mathrm{AU})$ & $\Delta x$ & 50.3465 & 50.3465 & 50.3465 & 50.3465 \\
\hline \multicolumn{7}{|c|}{ Simulation Outcomes } \\
\hline Final simulation time & (kyr) & $t_{\text {final }}$ & 179.3 & 232.2 & 42.8 & 49.6 \\
\hline Number of sink particles formed & & $n_{\text {sinks }}$ & 16 & 6 & 45 & 3 \\
\hline Max sink mass & $\left(M_{\odot}\right)$ & $\cdots$ & 2.01528 & 9.53198 & 19.4442 & 31.2937 \\
\hline Min sink mass & $\left(M_{\odot}\right)$ & $\cdots$ & 0.0264274 & 0.164572 & 0.00759937 & 8.42947 \\
\hline Mean sink mass & $\left(M_{\odot}\right)$ & $\cdots$ & 0.696485 & 2.84511 & 0.680372 & 19.8616 \\
\hline Median sink mass & $\left(M_{\odot}\right)$ & $\cdots$ & 0.525414 & 1.56426 & 0.0548092 & 19.8616 \\
\hline
\end{tabular}

material accumulates into filamentary structures quite quickly (top and middle panel from left to right), and that having an initially higher density more rapidly leads to dense filamentary structures due to the increased importance of gravity (bottom row, left and middle panels). Finally, the presence of a magnetic field acts to slow the accumulation of material into dense filaments, as can be seen comparing the top and middle row panels, or the bottom row left and middle panels. We will return to this point in more detail in Section 3 and beyond.

To extract the filamentary structure evident in Figure 1, we use the DISPERSE filament-finding algorithm ${ }^{6}$ described in Sousbie (2011) and Sousbie et al. (2011). The DisPerSE algorithm identifies persistent topological structures such as peaks, voids, and filaments, and is effective even if the image is noisy. It has been extensively used on Herschel observations for filamentary structure identification, e.g., Arzoumanian et al. (2011), Schneider et al. (2012), and Palmeirim et al. (2013). In DISPERSE, there are several user-defined parameters to control the resulting filamentary network: persistence and robustness thresholds, smoothing, and a maximum angle. The two thresholds can be thought of as very roughly corresponding

6 http://www2.iap.fr/users/sousbie/ to criteria for a minimum absolute brightness (persistence threshold) and a minimum relative brightness compared to neighboring features (robustness threshold). Smoothing removes small-scale "wiggles" from the initial filament spine, while the angle is used to specify the minimum angular rotation between two initial filament spine segments that can be joined together and still be classified as the same filament. Filament spine segments which meet at a right angle, for example, are likely not part of the same filament.

We identify filaments using a persistence threshold of 0.025 $\mathrm{g} \mathrm{cm}^{-2}$ and a robustness threshold of $0.05 \mathrm{~g} \mathrm{~cm}^{-2}$ in the $500 M_{\odot}$ simulation (or 7 and $14 \times 10^{21} \mathrm{~cm}^{-2}$ ) and thresholds of $0.1 \mathrm{~g}$ $\mathrm{cm}^{-2}$ and $0.2 \mathrm{~g} \mathrm{~cm}^{-2}$ (or 2.8 and $5.6 \times 10^{22} \mathrm{~cm}^{-2}$ ), respectively for the $2000 M_{\odot}$ simulation, smoothing the resulting filaments 1000 times, and allowing the initially identified filament segments to be connected for angles of less than $60^{\circ}$ (relative to a straight line). These parameters were chosen after testing a range of values to determine which values produced a filamentary structure that best matched visually apparent structures. All of these thresholds for DISPERSE are above the standard "threshold for star formation" found in nearby molecular clouds of around $\sim 5-7 \times 10^{21} \mathrm{~cm}^{-2}$ (e.g., Johnstone et al. 2004; Könyves et al. 2013). Unlike the 


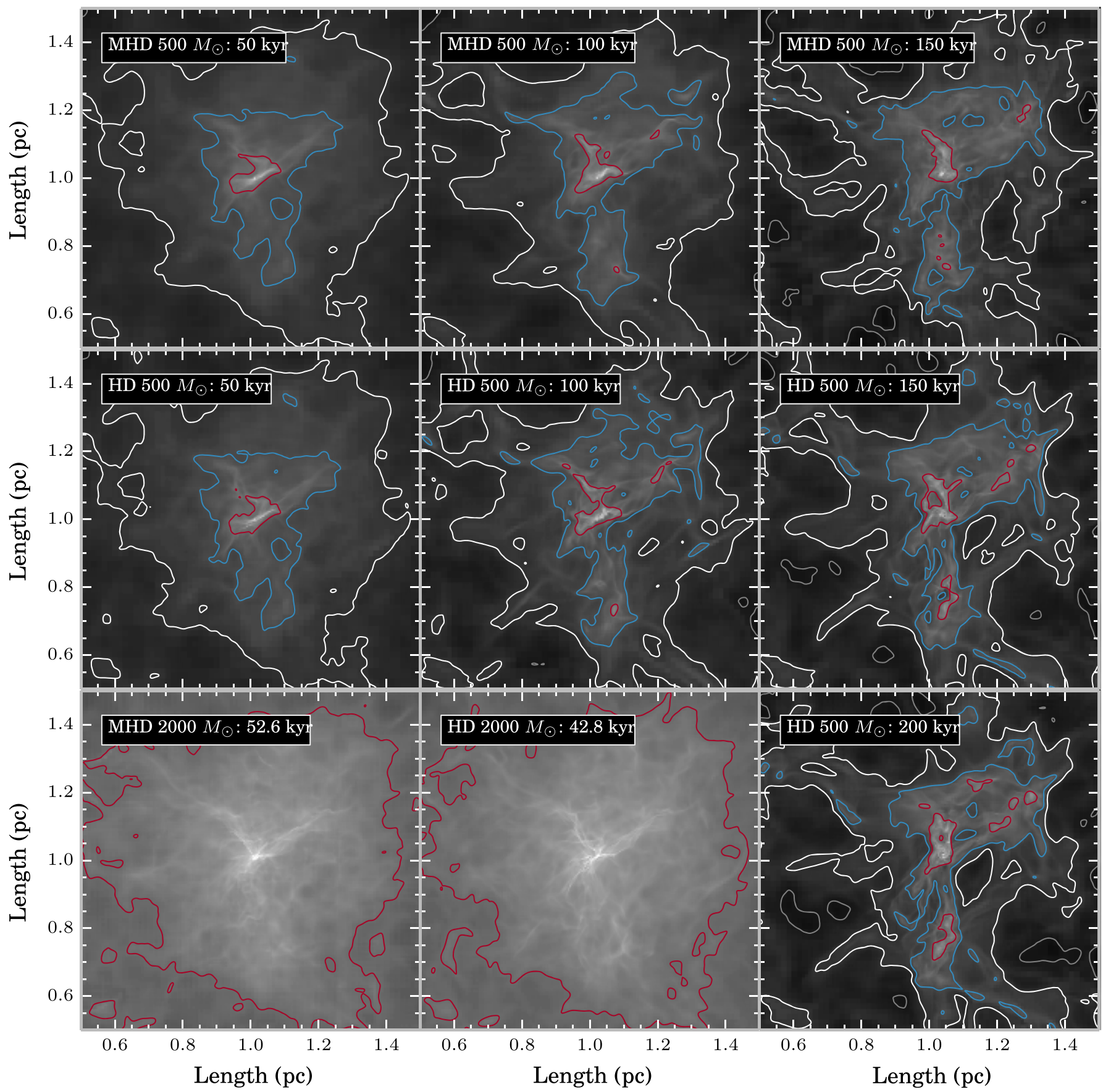

Figure 1. A comparison of the column density distribution projected along the $X$ axis for the simulations. The top two rows show the $500 M_{\odot}$ simulations at $0.05,0.1$, and $0.15 \mathrm{Myr}$ after the start of the simulation (left to right) for the MHD (top) and HD (middle) runs. The bottom row shows the MHD and HD $2000 M_{\odot}$ simulations at $0.05 \mathrm{Myr}$ (left and middle) and the $\mathrm{HD} 500 M_{\odot}$ simulation at $0.2 \mathrm{Myr}$ (right). All simulations are shown cropped to the inner $1.5 \mathrm{pc}$ to better show the smaller-scale structure that forms. The grayscale range is the same in all panels, going from 0.01 to $10 \mathrm{~g} \mathrm{~cm}^{-2}\left(\sim 3 \times 10^{21}\right.$ to $\left.3 \times 10^{23} \mathrm{~cm}^{-2}\right)$ from black to white, with a logarithmic scaling applied. The overlaid contours show column densities of $0.02,0.04,0.075$, and $0.2 \mathrm{~g} \mathrm{~cm}^{-2}\left(5.3,1.1,2.1\right.$, and $\left.53 \times 10^{21} \mathrm{~cm}^{-2}\right)$ in gray, white, blue, and red, respectively. If several assumptions are made, including that all pixels belong to cylindrical structures with a characteristic width of $0.1 \mathrm{pc}$, then the contours also correspond to mass per unit length values $0.5,1,2$, and 5 times the critical mass per unit length at a temperature of $10 \mathrm{~K}\left(18 M_{\odot} \mathrm{pc}^{-2}\right)$. Note that these assumptions are poor for regions not associated with filamentary structure. See Section 3.4 for more detail.

Herschel analyses, we applied DisPeRSE directly on the column density map. Since our column density maps include only the gas from the simulated star-forming clump, with no potential contribution from other dense structures within the larger cloud, we have less need than with Herschel data to apply filament-enhancing algorithms. Finally, we excluded several very short filaments that DISPERSE initially identified- in order to accurately determine the filament profile (below), we set a minimum length of $0.1 \mathrm{pc}$.

Figure 2 shows the network of filaments identified in the $\mathrm{X}$ projection of the $500 M_{\odot}$ and $2000 M_{\odot} \mathrm{HD}$ simulations overlaid on their column density maps.

One of the goals of our analysis is to track the time evolution of and the effect of magnetic fields on individual filaments. In 

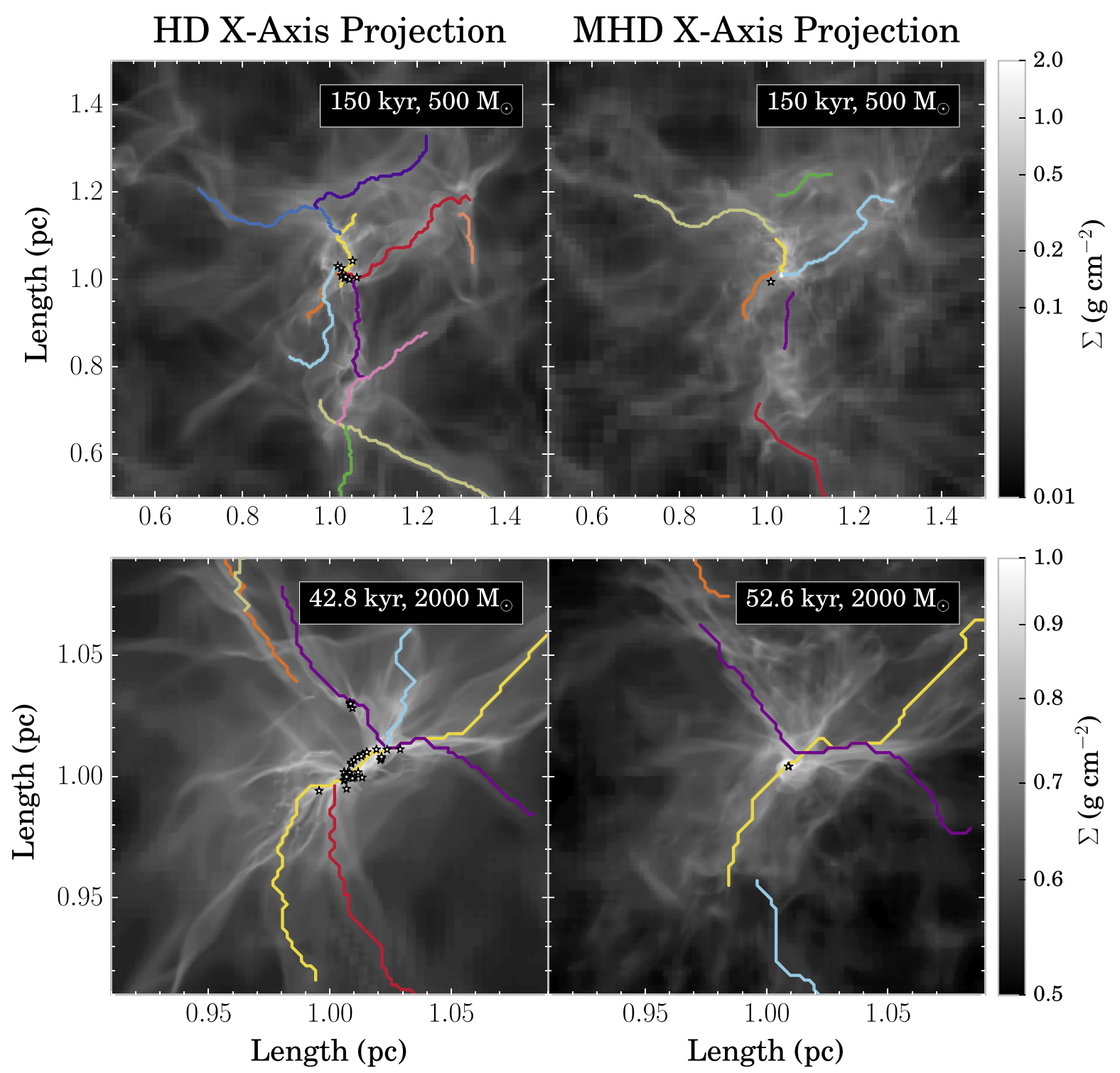

Figure 2. Examples of the filamentary structure identified in the simulations. The top panel shows the filaments identified using DisPeRSE in the X projection of the $500 M_{\odot} \mathrm{HD}$ (left) and MHD (right) simulations at $0.15 \mathrm{Myr}$, while the bottom panel similarly shows the $2000 M_{\odot} \mathrm{HD}$ (left) and MHD (right) simulations at $0.05 \mathrm{Myr}$. Each colored line indicates a unique filament spine identified in that projection. Note that the top and bottom panels zoom in to different extents to best illustrate the central filamentary structure; similarly, each row has a different grayscale scaling applied—see the scalebar on the right hand side. In all panels, sink particles formed at the specified time are shown by the white stars; in all cases, their formation is confined to the central clustered part of the simulation.

order to do so, DisPerSE was not used to identify a different network of filaments at every time step and magnetic field value, as this could potentially lead to different filaments being identified at different snapshots. Instead, for each of the three projections, we started with the network of filaments identified with DisPerSE at $0.15 \mathrm{Myr}$ in the HD simulation, and then searched for the corresponding structures at different times and with magnetic fields present. For the $2000 M_{\odot}$ simulations, we instead started with the single $0.05 \mathrm{Myr}$ time step. We started with an automated procedure to identify equivalent filaments at other time steps and/or with magnetic fields, by searching for local column density maxima near the reference set of filament spines. After this step, all filament spines were verified and adjusted as necessary by hand, using a combination of visual inspection of the current column density snapshot and a movie of the time evolution of the column density map for the $500 M_{\odot}$ simulations. The simulations, particularly without the moderating presence of magnetic fields, form significant substructure on all scales, making it difficult to impossible for an automated procedure to correctly "follow" the filaments in time and across initial conditions.

There are several cases where a filament could not be fully traced to earlier times or in the corresponding simulation with magnetic fields. Some, but not all, of these cases appear to be attributable to structures which are only apparent as filaments in 2D due to a coincidence of independent 3D structures; at other time steps, the real 3D structures have moved by different amounts and no longer appear connected. We include these structures in our analysis where they do appear as a single filamentary structure, as any real observation which only has column density information is fallible to the same line of sight coincidence confusion. We will address the full 3D nature of filaments in these simulations in an upcoming paper. 
A comparison of Figures 1 and 2 shows that the filamentary network identified lies only in the very densest part of the cloud, where the estimated mass per unit length value is signficantly above the thermal critical value (white contours in Figure 1). We will return to this point further in Sections 3.2 and 3.3 .

\subsection{Calculating Radial Column Density Profiles}

Once the filaments are identified, we measure the radial column density profiles along them. Since the filaments tend to converge toward the simulation center, and sometimes even intersect, care is needed to properly calculate the radial column density profile. First, we assign every pixel to the filament to which it is closest. Next, we exclude pixels which lie very close $(<0.01 \mathrm{pc})$ to two or more filaments - this value was chosen to provide a balance between not including too many locations which might provide non-representative measures of a given filament profile, and not excluding too large a fraction of material around the filaments. We then calculate the mean column density of pixels in separation bins equal to the pixel size $(\sim 0.002 \mathrm{pc})$. Finally, to ensure that the filament profiles are accurate, we exclude the measurement for any radial bin where at least $25 \%$ of the total length of the filament, at that separation, was not included in the profile calculation. This final criterion ensures that all radial column density profile measurements used in our analysis are reliable-there are no cases where data from only a few pixels are used to infer the filament's properties. We note that the above restrictions limit our analysis to a smaller range of radii than used in Smith et al. (2014), although the range is closer to Arzoumanian et al. (2011). Smith et al. (2014) analyze only the brightest one or two filaments in any given simulation snapshot, which ensures that the contamination in filament profiles will be minimal; with our inclusion of fainter filaments, only smaller radial separations from a given filament spine are free from material from neighboring filaments. Figures 3 and 4 show several example radial column density profiles which will be further discussed in Section 4.

\section{BASIC FILAMENT PROPERTIES}

Visual inspection of the resulting radial column density profiles (e.g., Figures 3 and 4) reveals a variety of characteristics. We expect that after the first turbulent shocks form a filamentary structure, gravity acts to continue to concentrate mass onto these filaments, leading to higher and narrower peaks with time. An initially higher mean density should increase gravity's pull and lead to a faster filament evolution. The presence of a magnetic field should cushion the initial turbulent compressions, reducing the amount of material initially in the filament, and giving the appearance of "fluffier" filaments. The subsequent evolution of MHD filaments should then be slowed relative to the HD case by gravity's weaker pull on the the initial lower concentration of mass, and possibly also further action by the magnetic field, depending on its orientation.

Broadly, these behaviors do hold-the visual impression from watching movies of each simulation suggest this behavior; nevertheless, we find instances of filaments dissipating over time, suggesting gravity was insufficient to prevent the initial turbulent compression from re-expansion. In some of these cases, magnetic fields appear to help to slow or prevent

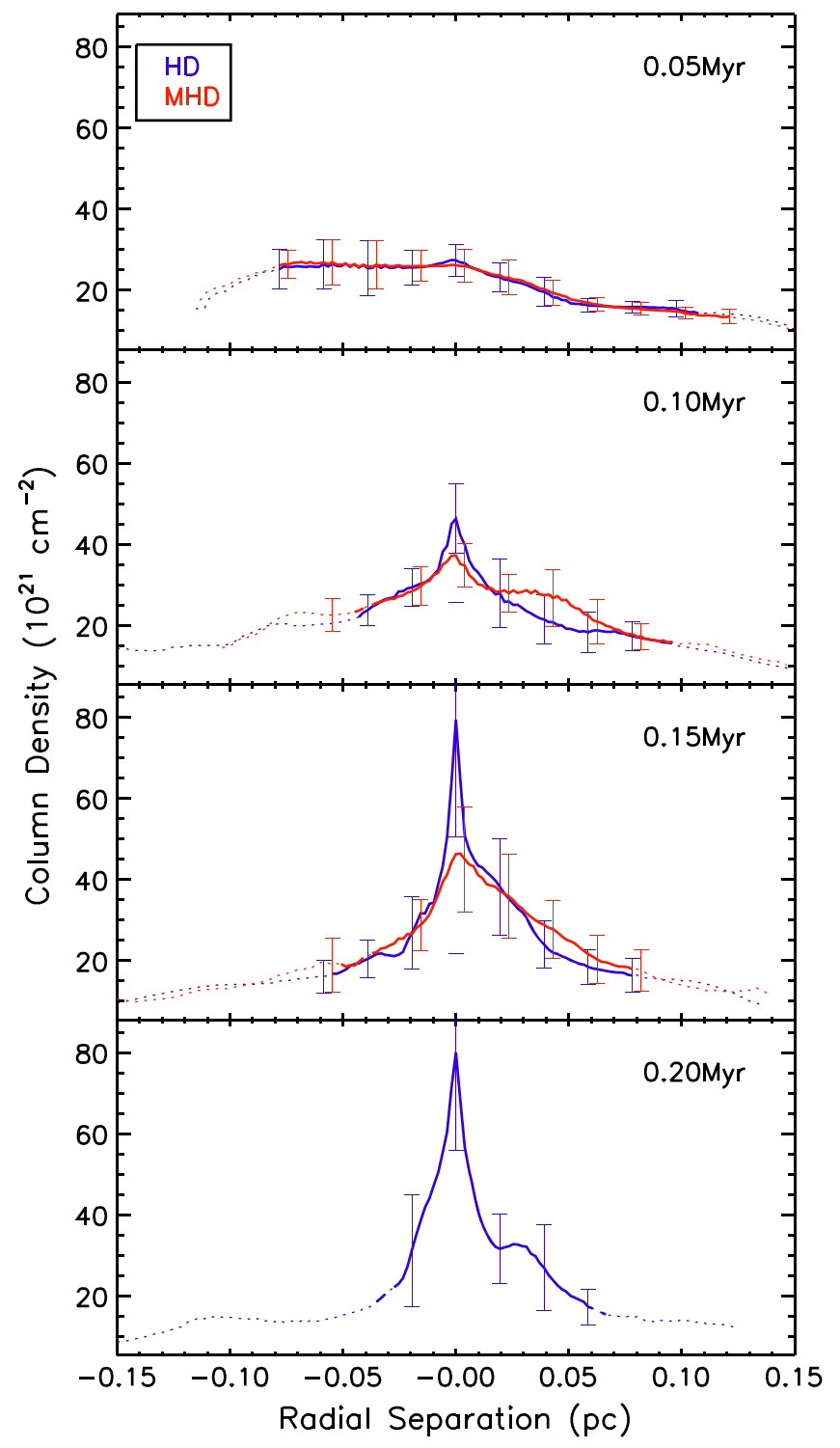

Figure 3. Time evolution of the radial column density profile of a filament identified in the $\mathrm{Z}$ projection of the $500 M_{\odot}$ simulations. Solid lines show the reliable portion of the column density profile, while the dotted lines indicate less reliable measurements (i.e., data over less than $75 \%$ of the length of the filament was included). The error bars indicate the standard deviation of column density values at that radial separation. The MHD error bars are slightly shifted for better legibility. In this example, the filament continues to contract in both the MHD and HD simulations, although at a faster rate in the HD case.

this re-expansion, causing the HD filament to have a higher and narrower peaked profile than in the MHD case. In other instances, data excluded for one or more of the reasons mentioned above (difficulty in tracing the filament, or exclusion due to unreliability) also prevents the full influence of time or magnetic fields to be fully assessed.

Despite this more complex behavior, there are still several simple measures that we can make to gain insight into the behavior observed.

\subsection{Filament Widths}

The conceptually simplest measureable filament property is its width. Although filament widths measured with Herschel span at least a factor of five (e.g., Figure 7 in Arzoumanian et 


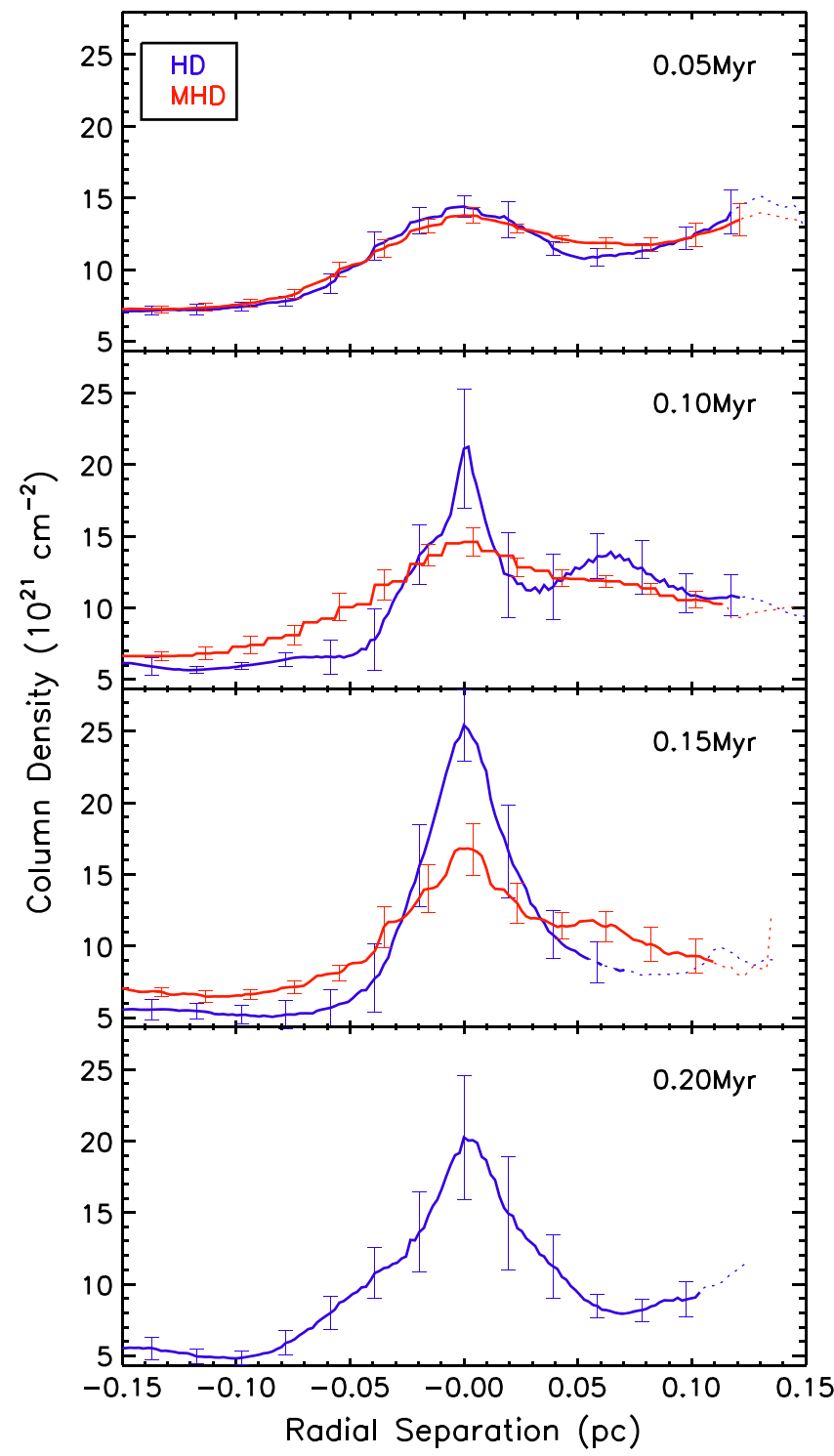

Figure 4. Time evolution of the radial column denstiy profile identified in the $\mathrm{Z}$ projection of the $500 M_{\odot}$ simulations. See Figure 3 for details on the plotting conventions used. In this example, the filament contracts and then expands in both the HD and MHD simulations.

al. 2011), it is often stated that filaments have a constant width of $\sim 0.1$ pc. Note, however, that Juvela et al. (2012a) find a larger scatter in filament FWHM values in their analysis of (different) Herschel data, although some of their filaments are much more massive and/or more distant than the Arzoumanian et al. (2011) sample. We measure the width of all of the filaments tracked in our simulations in the simplest possible method-the extent of the radial profile at half of the peak value, i.e., the FWHM. Table 2 shows our results, separated by time step, magnetic field, and mass. Included is the mean and standard deviation of FWHM values measured, along with the number of FWHM values considered. Some filaments did not have reliable radial column density profiles out to sufficiently large radial separations to allow the FWHM to be measured; these were excluded from the values given in Table 2 .

Although the dispersion is large, owing in part to the disparate behaviors discussed earlier, it is clear that on average, the filaments do behave as expected. Filaments generally get narrower with time and in higher mean density environments, and are wider when magnetic fields are present. Furthermore, this trend is somewhat subtle: all of the simulation snapshots give filaments that have widths within the range that observers find. Heitsch $(2013 a, 2013 b)$ notes that in accreting filament models, the filament width can remain relatively constant throughout much of a filament's evolution, if either the ram pressure from accreting material is small (Heitsch 2013a) or in the case of a weakly magnetized accretion. Hennebelle \& André (2013) propose a model wherein ion-neutral friction dominating the dissipation of turbulence accounts for a relatively constant filament width of $\sim 0.1 \mathrm{pc}$ while Gómez \& Vázquez-Semadeni (2014) suggest a constant width may be caused by a balance between large-scale accretion onto filaments and accretion from the filaments onto the dense cores and stars forming within them. Our simulations do not contain ambipolar diffusive effects for the magnetic field, so that the substructure formed involves a balance between accretion, gravity, and turbulence. Future work will measure the accretion onto filaments versus the dense cores.

We also tried fitting Gaussians to the filament profiles, with a constant background level set as a free parameter (fits not shown), similar to other works (Arzoumanian et al. 2011; Smith et al. 2014). ${ }^{7}$ Table 3 shows the equivalent FWHMs derived from the Gaussian fits for the filaments. Although the FWHM values tend to be smaller when using a Gaussian fit with a non-zero background, especially at earlier times (where the relative amplitude of the background is larger), the same general trends hold true: the filament width decreases with time and tends to be larger when magnetic fields are present. The mean widths are still generally consistent with the observations, although the range of widths is larger in the simulations, similar to the findings of Smith et al. (2014).

\subsubsection{Biases}

Smith et al. (2014) provide a detailed consideration of factors which can impact the measured filament width. For example, they find that when performing a Gaussian fit, the best-fit FWHM strongly depends on the radial extent of the profile: including measurements from larger separations from the filament spine tends to increase the FWHM. Presumably this is at least partly caused by a lower background column density being fit for profiles that extend further from the filament spine. Our analysis tends to use a smaller radial extent than in Arzoumanian et al. (2011) and especially Smith et al. (2014) due to potential contamination from other nearby filaments, which may explain why we tend to measure narrower filament widths in our Gaussian fits. ${ }^{8}$ Measuring the FWHM directly from the profile will be robust to radial range variations, but has its own bias. Unresolved central filament peaks become lower with poorer resolution, which would change the peak column density used to estimate the FWHM (R. Smith 2015, private communication). Both the biases in the FWHM and Gaussian-fitted width measurements are primarily systematic, affecting absolute rather than relative values. (We emphasize that this statement does not imply that the range in

\footnotetext{
7 All of our fits (including those in Section 4) make use of the IDL mpfit routine by Markwardt (2009), available at http://physics.wisc.edu/ craigm/idl/ fitting.html.

8 Smith et al. (2014) focus their analysis on the brightest one or two filaments in each of their simulations, which ensures that the contamination from other filamentary structures will be more minimal, even with a larger radial extent.
} 
Table 2

Filament FWHM Values

\begin{tabular}{|c|c|c|c|c|c|c|c|c|c|}
\hline $\begin{array}{l}\text { Mass } \\
\left(M_{\odot}\right) \\
\end{array}$ & $\begin{array}{l}\text { Time } \\
(\mathrm{Myr})\end{array}$ & \multicolumn{2}{|c|}{ HD_FWHM Stats ${ }^{\mathrm{a}}$} & \multicolumn{2}{|c|}{ MHD_FWHM Stats ${ }^{\mathrm{a}}$} & \multicolumn{2}{|c|}{ HD—FWHM Stats ${ }^{\text {b }}$} & \multicolumn{2}{|c|}{ MHD—FWHM Stats ${ }^{\mathrm{b}}$} \\
\hline 500 & 0.05 & 0.212 & 0.132 & 0.262 & 0.164 & 0.211 & 0.127 & 0.268 & 0.175 \\
\hline 500 & 0.15 & 0.079 & 0.073 & 0.130 & 0.090 & 0.050 & 0.028 & 0.104 & 0.051 \\
\hline 500 & 0.20 & 0.058 & 0.047 & N/A & N/A & 0.044 & 0.025 & N/A & N/A \\
\hline 2000 & 0.05 & 0.119 & 0.113 & 0.168 & 0.147 & 0.132 & 0.137 & 0.170 & 0.142 \\
\hline
\end{tabular}

Notes.

${ }^{\text {a }}$ All measureable filaments included.

${ }^{\mathrm{b}}$ Only filaments where the FWHM could be measured at all times in HD and MHD were included. Note that the $500 M_{\odot}$ and $2000 M_{\odot}$ filaments are different samples.

Table 3

Filament Gaussian-fit FWHM Values

\begin{tabular}{|c|c|c|c|c|c|c|c|c|c|}
\hline $\begin{array}{l}\text { Mass } \\
\left(M_{\odot}\right) \\
\end{array}$ & $\begin{array}{l}\text { Time } \\
\text { (Myr) }\end{array}$ & \multicolumn{2}{|c|}{ HD_FWHM Stats ${ }^{\mathrm{a}}$} & \multicolumn{2}{|c|}{ MHD_FWHM Stats ${ }^{\mathrm{a}}$} & \multicolumn{2}{|c|}{ HD_FWHM Stats ${ }^{\mathrm{b}}$} & \multicolumn{2}{|c|}{ "MHD-FWHM Stats ${ }^{\mathrm{b}}$} \\
\hline 500 & 0.05 & 0.08 & 0.07 & 0.10 & 0.07 & 0.11 & 0.10 & 0.12 & 0.09 \\
\hline 500 & 0.15 & 0.04 & 0.02 & 0.06 & 0.03 & 0.05 & 0.02 & 0.07 & 0.03 \\
\hline 500 & 0.20 & 0.05 & 0.02 & N/A & N/A & 0.05 & 0.02 & N/A & N/A \\
\hline 2000 & 0.05 & 0.04 & 0.03 & 0.10 & 0.11 & 0.05 & 0.03 & 0.15 & 0.15 \\
\hline
\end{tabular}

Notes.

a All measureable filaments included.

${ }^{\mathrm{b}}$ Only filaments where a Gaussian could be fit at all times in HD and MHD were included. Note that the $500 M_{\odot}$ and $2000 M_{\odot}$ filaments are different samples.

widths is invariant, but that the relative rankings likely are, i.e., the widest filaments appear to be the widest with any measure.) We expect then that our conclusions about the weak trends in width are therefore robust, a point supported by the similarity in behavior using either width measurement.

Finally, we note that the timescale over which we are able to analyze the filaments is relatively short: 0.2 to 0.3 times the global free-fall time for the $500 M_{\odot}$ simulations, and 0.13 times the global free-fall time for the $2000 M_{\odot}$ simulations. Since the filaments form in the denser parts of the simulation, a larger number of local free-fall times would have elapsed. Nonetheless, analysis over a longer timescale could reveal stronger signs of filament evolution than we are able to probe here.

\subsection{Mass per Unit Length}

The simplest equilibrium model for a filament is that of the isothermal cylinder, presented in Ostriker (1964), where gravity is balanced by thermal pressure along an infinite cylinder. In this model, the stability of the cylinder is controlled by the mass per unit length, $M_{\text {line }}$. The critical mass per unit length, in turn, depends only on the temperature:

$$
M_{\text {line }}^{\text {crit }}=\frac{2 k_{B} T}{\mu m_{H} G} \equiv \frac{2 c_{s}^{2}}{G},
$$

where $c_{s}$ is the sound speed and $G$ the gravitational constant (Ostriker 1964). Furthermore, Inutsuka \& Miyama (1997) showed that isothermal filaments are unstable to axisymmetric perturbations of wavelength greater than about 2 times the filament diameter if the mass per unit length is close to this critical value.
In our simulations, the temperature is set at a constant $10 \mathrm{~K}$, which implies $M_{\text {line }}^{\text {crit }}=18 M_{\odot} \mathrm{pc}^{-1}$. Turbulent motions can also provide additional support through raising the typical velocity dispersion of the gas above the thermal value; Heitsch (2013a) points out that non-thermal motions can be driven by the accretion of material onto the filament itself (see also Peretto \& Fuller 2014). Fiege \& Pudritz (2000) show that a more appropriate critical mass per unit length value is given by

$$
M_{\mathrm{line}}^{\text {crit }}=\frac{2\left\langle\sigma^{2}\right\rangle}{G},
$$

where $\left\langle\sigma^{2}\right\rangle$ is the velocity dispersion including both thermal and non-thermal components. A careful analysis of the velocity fields would be required to determine precisely how much nonthermal support is provided on the scales of interest; the approximation often assumed is

$$
\sigma^{2}=c_{s}^{2} \times\left(1+\mathcal{M}^{2} / 3\right)
$$

(e.g., Klessen et al. 2000). With $\mathcal{M} \sim 6$ in our simulations, that would lead to raising $M_{\text {line }}^{\text {crit }}$ by a factor of roughly 37 , giving 670 $M_{\odot} \mathrm{pc}^{-1}$.

In the case of a magnetized turbulent cloud, there is a magnetic correction that must be made to Equation 3. In the case where there is only a poloidal magnetic field and no toroidal (wrapped field) component, the magnetic pressure helps support the filament against gravity. Taking Equation (27) of Fiege \& Pudritz (2000) with $\Gamma_{\phi}=0$ (no toroidal field), and using Fiege \& Pudritz's (2000) Equation (23) to convert between the vertical magnetic field flux and magnetic field 
strength, the critical mass per unit length becomes

$$
M_{\text {line }}^{\text {(crit, } B)}=M_{\text {line }}^{\text {crit }} \times\left(1+\mathcal{M}_{A}^{2} / 2\right)
$$

where $\mathcal{M}_{A}=v_{A} / \sigma$ is the turbulent Alfvén Mach number whose Alfvén speed, $v_{A}$, is $B / \sqrt{4 \pi \rho}$. For super-Alfvénic turbulence, $\mathcal{M}_{A}<1$, this correction is slight. In our simulation, however, $\mathcal{M}_{A}$ is 2.1-2.2 (see Table 1). Thus, our MHD turbulence is somewhat sub-Alfvénic, i.e., the magnetic field strength dominates the turbulence, and this implies that the critical line mass for our models is somewhat greater than the purely hydrodynamic turbulent case; $M_{\text {line }}^{\text {(crit, } B)} \sim 3 M_{\text {line }}^{\text {crit }}$. This result predicts that our MHD case should be considerably less susceptible to fragmentation than the hydro case. We note that as the turbulence is damped and the line width reduces to the thermal value, the relative magnetic contribution to the (thermal) line mass can become significantly more important depending on the orientation of the field across the filament.

There has been little time in these simulations for the turbulence to decay, but some of the power of the turbulence is on larger scales than our filaments, so the turbulent critical mass per unit length of $670 M_{\odot} \mathrm{pc}^{-1}$ is an upper limit to the true effective critical mass per unit length of the simulated filaments. Indeed, the observed velocity dispersion in filamentary gas tends to be only of order twice the sound speed in nearby filaments that have peak column densities similar to those formed in our simulations (e.g., Hacar \& Tafalla 2011; Arzoumanian et al. 2013; Hacar et al. 2013; Kirk et al. 2013). Arzoumanian et al. (2013) furthermore find that for filaments with masses per unit length much higher than the critical thermal mass per unit length, the velocity dispersion increases with increasing mass per unit length, a trait they attribute to infall of material onto the filaments. In our simulations, most if not all of the turbulent motion is likely due to the remnants of the initial turbulence, given there has been little time for that to decay, or for infall to generate additional turbulent motions. Assuming the total filament velocity dispersion is twice the thermal value would increase $M_{\text {line }}^{\text {crit }}$ by a factor of four. The estimated mass per unit length contours shown on Figure 1 illustrate that most of the dense filamentary structure is found in areas with $M_{\text {line }}^{\text {crit }}$ above $4-5$ for the $500 M_{\odot}$ simulations, and even higher in the $2000 M_{\odot}$ simulations. Peretto \& Fuller (2014) estimated that non-thermal support in SDC13 would contribute to lowering the mass per unit length values in the filaments to 1-2 times the critical value from 4-8 times the critical value if non-thermal motions were not accounted for. Note that depending on the orientation, magnetic fields could either aid or hinder gravitational collapse.

The degree of gravitational fragmentation of the filaments can, to some degree, be ascertained by the number of sink particles that form in the simulations. It is notable that the sinks are typically found in or near the filaments. It is also clear that the number of sinks that form in MHD simulations is smaller, sometimes notably so, in comparison with their hydrodynamic counterparts. Thus, using the data in Table 1, we see that in the $500 M_{\odot}$ simulation, while 16 sinks particles appear in the HD run, only 6 are apparent in the MHD case. The suppression is even greater in the $2000 M_{\odot}$ simulation (although in that case the runtime was shorter) where 45 formed in the HD case as compared to 3 in the MHD case. Clearly, magnetic support is significantly reducing fragmentation.

Non-isothermal equilibrium cylinder models have also been investigated (see Recchi et al. 2013 and the discussion therein).
In the case of a thermally supported equilibrium cylinder, where the temperature gradually increases outward, similar to observations, Recchi et al. (2013) find that the mass per unit length that can be supported is only about $20-30 \%$ larger than in the isothermal case. Since the simulations we analyze are strictly isothermal, we do not consider this class of models further here.

\subsection{Individual Filament $M / L$ Measurements}

Measuring the total mass of each filament is difficult as can be seen in Figures 1 and 2; the filaments do not tend to have clearly defined outer boundaries. This challenge is exacerbated by the fact that many of the filaments lie close to one anotherthe total mass cannot be derived by including material arbitrarily far away from the filament's spine. We determined that the best way to estimate each filament's mass was to include only material within the FWHM of the filament spine. While this will necessarily provide a lower limit to the true filament mass, lower and wider thresholds, such as the full width at quarter maximum, cannot be determined for too large a fraction of the total filament population (see discussion above). Using a constant width for the mass determination would bias the estimates toward relatively lower values for the wider/fluffier filaments. We note that instead of adopting a filament width based on the Gaussian fits discussed in Section 3 yields a similar behavior to that discussed below.

Using the estimated filament mass per unit lengths, we can test whether this metric is a useful predictor of filament stability. A very simple proxy for the evolutionary path of a filament is to compare the peak column density (in the radial column density profile) at two neighboring time steps. If the filament is contracting or accreting, the second peak should be higher than the first, while the reverse would be true for an expanding filament. ${ }^{9}$ We would therefore expect that higher mass per unit length values (above the critical value) would correspond to a ratio in peak column densities of greater than one (for the later time divided by the earlier time).

Figure 5 shows the mass per unit length of each filament compared to the ratio of the peak column density at the subsequent and current time steps. The thermal critical mass per unit length for a temperature of $10 \mathrm{~K}$ is $\sim 18 M_{\odot} \mathrm{pc}^{-1}$, below nearly all of our measurements. Non-thermal motions likely contribute some amount of support (Section 3.2). In Figure 5 we show the critical mass per unit length assuming the total velocity dispersion is twice the thermal value, which gives a value of $\sim 72 M_{\odot} \mathrm{pc}^{-1}$. While this is a rough approximation, it appears to denote the level above which no filaments are found to be expanding. Regardless of precisely where the effective critical mass per unit length is drawn, we note a surprising result: many points occupy the bottom right quadrant, contracting filaments whose current mass per unit length implies gravitational stability. Similarly, there are several filaments whose mass per unit length ratio suggests graviational instability that are instead expanding. Although some of the presently contracting, low mass per unit length filaments (bottom right) may expand at time steps beyond what we can

\footnotetext{
9 We verified this assumption by comparing the ratio of filament FWHM values at subsequent times and found very good correlation: over $86 \%$ of filaments interpreted as contracting or expanding based on their peak column density ratio at neighboring time steps show the same signature in their FWHM ratio. Allowing for slight measurement uncertainties (5\% error in the ratios) gives an agreement between the two ratio measurements of just over $95 \%$.
} 


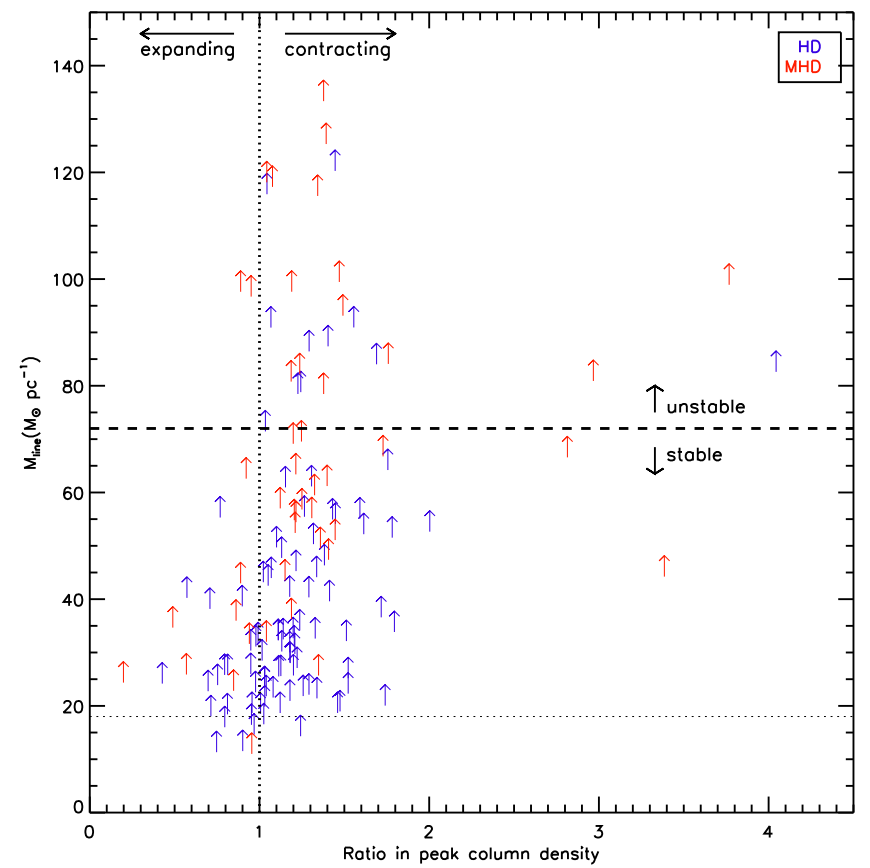

Figure 5. The mass per unit length measured for a filament at a given time step vs. the ratio in peak column densities for the subsequent vs. current time step. Filaments which are contracting would be expected to have a ratio in peaks greater than one (right of the vertical dotted line), whereas filaments which are re-expanding would have a ratio in peaks of less than one (left of the vertical line). The thick horizontal dashed line indicates the estimated effective mass per unit length (assuming equal thermal and non-thermal contributions); filaments with values above this line would be expected to be contracting due to gravity, whereas those with lower values are stable against gravitational collapse. The thin horizontal dotted line indicates the critical mass per unit length assuming just thermal support; nearly all of the filaments have estimated mass per unit length values well in excess of the thermal critical value. Note that the estimated mass per unit length values are all lower limits (see text for details).

trace, the figure highlights the fact that predictions about the future evolution of filaments are incomplete when only column density information is available. This is also apparent in Figure 6, where the filament with the initially higher peak column density is the one which later re-expands. Although the individual mass per unit length values are not a good predictor of future evolution, the fact that there is a weak correlation between mass per unit length and peak column density ratio suggests that some (limited) insight into the bulk behavior of filaments can be gained from the simple isothermal-withturbulence model.

\subsection{Region-wide Mass Per Unit Length}

Finally, we can also get a rough idea of the stability of all the material in the simulation. The Herschel Gould Belt team has provided an estimate of the mass per unit length at every pixel, for material in their curvelet map, i.e., that associated with structures having long axis ratios (e.g., André et al. 2010). These mass per unit length estimates are made under the assumption that each pixel is part of a filament or cylindrical structure with a typical width of $0.1 \mathrm{pc}$. The contours in Figure 1 can be interpreted under a similar set of assumptions, although we emphasize that since our calculation includes the entire mass in the simulation, the equivalent mass per unit length values should not be over-interpreted in regions not associated with filamentary structure. The thermal critical value

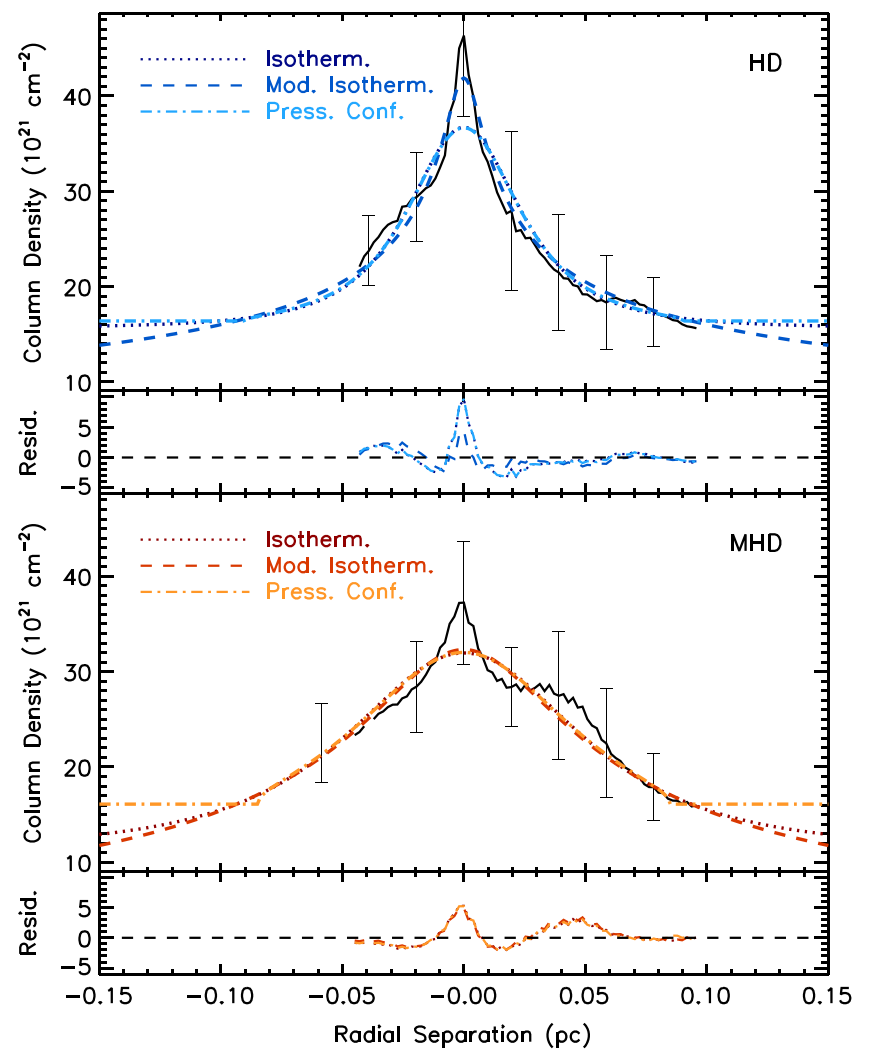

Figure 6. The radial column density profile for the filament in Figure 3 at 0.1 Myr, including the best-fit models with a background column density term. The top panel shows the profile and fits for the HD simulation, as well as the residuals between the models and simulation, while the bottom panel shows the same for the MHD simulation. The solid black line indicates the mean column density at each radial separation, while the error bars denote the standard deviation in values at various radial separations. The model lines shown are the purely isothermal cylinder (darkest, dotted line), the modified isothermal cylinder (less dark, dashed line), and the pressure-confined cylinder (lightest, dashed-dotted line).

assumed for the contours in Figure 1 is that for a $10 \mathrm{~K}$ medium, i.e., $M_{\text {line }}^{\text {crit }}=18 M_{\odot} \mathrm{pc}^{-1}$. Most of the mass in filaments in the simulation lies above the critical mass per unit length value; if thermal pressure was the only source of support preventing gravitational collapse, we would expect to find sink particles forming throughout the simulation. Instead, all of the sink particles form at mass per unit length values of at least 5 (red contour in Figure 1). Including nonthermal motions, as discussed earlier, raises the critical mass per unit length, therefore decreasing the ratio of the mass per unit length to the critical value. The mass per unit length of individual filaments above which only contracting filaments are found, as discussed in Section 3.3, was a similar factor (4) above the thermal critical value. It therefore appears that only the densest parts of the simulation, where filaments are present, are likely to be sufficiently dense for gravitational collapse to occur.

\section{MODEL COMPARISONS}

We next compare the radial column density profiles obtained to several cylindrical equilibrium models: the isothermal cylinder, modified isothermal cylinder, and pressure-confined isothermal cylinder, described in detail below. In the simplest model, the isothermal cylinder, thermal pressure balances gravity along an infinite cylinder, leading to a $3 \mathrm{D}$ density 
profile decreasing as $r^{-4}$ at large radii, or the column density varying as $r^{-3}$ (Ostriker 1964). Herschel teams have found that the observed column density profiles are shallower than the isothermal cylinder model and and instead use a modified profile, also referred to as a "Plummer-like" profile (Plummer 1911; Nutter et al. 2008; Smith et al. 2014), where the power law exponent is an additional fitted parameter:

$$
\Sigma(r)=A_{p} \frac{\rho_{c} R_{\mathrm{fl}}}{\left(1+\left(r / R_{\mathrm{fl}}\right)^{2}\right)^{\frac{p-1}{2}}} .
$$

Here, $\Sigma$ is the column density, $\rho_{c}$ is the central density, $R_{\mathrm{fl}}$ represents the scale of the inner flat portion of the profile, $p$ is the power law index (with a value of 4 for the original Ostriker model), and $A_{p}$ is a geometrical factor given by:

$$
A_{p}=\frac{1}{\cos i} \int_{-\infty}^{\infty} \frac{d u}{\left(1+u^{2}\right)^{p / 2}}
$$

where $i$ is the (unknown) inclination of the filament on the plane of the sky, assumed to be 0 (Arzoumanian et al. 2011). The best-fitting value of $p$ often tends to range between 1.5 and 2.5, a much shallower drop-off than in the $p=4$ Ostriker (1964) model (e.g., Alves et al. 1998; Lada et al. 1999; Arzoumanian et al. 2011; Hill et al. 2012; Juvela et al. 2012b; Malinen et al. 2012; Palmeirim et al. 2013). Some filaments, however, have been observed with column density profiles that are consistent with a $p=4$ isothermal model, (e.g., Nutter et al. 2008; Hacar \& Tafalla 2011; Pineda et al. 2011; Bourke et al. 2012), while Contreras et al. (2013) find $p=4$ provides a good fit around star-forming clumps and $p=2$ is better in the interclump areas. Theoretically, shallow radial column density profiles are consistent with equilibrium isothermal cylinder models that include helical magnetic fields (Fiege \& Pudritz 2000). Smith et al. (2014) show that $p \sim 2$ profiles are the norm for prominent filaments in hydrodynamic simulations without magnetic fields, regardless of the type of turbulence considered.

We also applied the equilibrium model of Fischera \& Martin (2012), in which pressure from the medium surrounding the filament is also included in the force balance. In this analytic formulation, the two quantities of interest are $P$, the pressure from the external medium, and $f$, the ratio of the mass per unit length to the critically stable value for the Ostriker cylinder. The full profile is given by:

$$
\begin{aligned}
& N_{H}(x)=\sqrt{\frac{P}{4 \pi G}} \frac{\sqrt{8}}{1-f}\left(\mu m_{H}\right)^{-1} \times \frac{1-f}{1-f+x^{2} f} \\
& \left(\sqrt{f(1-f)\left(1-x^{2}\right)}+\sqrt{\frac{1-f}{1-f\left(1-x^{2}\right)}}\right. \\
& \left.\times \arctan \sqrt{\frac{f\left(1-x^{2}\right)}{1-f\left(1-x^{2}\right)}}\right)
\end{aligned}
$$

where $N_{H}$ is the column density in number units, $x$ is a scaled radial coordinate, $G$ is the gravitational constant, $\mu$ is the mean molecular weight, and $m_{H}$ is the mass of a hydrogen atom (Fischera \& Martin 2012). The main effect of the pressure, $P$ is on the height of the central column density peak, while $f$ controls the shape of the profile (Fischera \& Martin 2012). (The temperature of the gas is also fit as part of the scale factor converting the radial coordinate $x$ into a physical radial separation.) In their re-analysis of the Herschel filaments in Polaris, IC 5146, and Aquila, Fischera \& Martin (2012) find that their pressure equilibrium model also provides a good fit to the filaments, with external pressures consistent with the range expected in the ISM.

In all of the models, a non-zero background column density can be included as a free parameter, i.e.,

$$
\Sigma(r)=\Sigma_{\text {model }}(r)+\Sigma_{0}
$$

where $\Sigma_{0}$ is a constant.

Most if not all of the filament analyses include a background column density term (e.g., Arzoumanian et al. 2011; Fischera \& Martin 2012; Juvela et al. 2012b; Palmeirim et al. 2013; Smith et al. 2014); Herschel analyses in fact allow the background to be fit by a linearly varying background column density: $\Sigma(r)=\Sigma_{\text {model }}(r)+\Sigma_{0}+\Sigma_{1}(r)$ (see Appendix B of Palmeirim et al. 2013), although the fits are generally similar when just a constant background column density is adopted (D. Arzoumanian 2015, private communication). We tried fitting the profiles with and without a background column density and found that including the background generally produced superior fits. In the case of the modified isothermal cylinder model (Equation (5)), including a background term decreased the central density, and allowed for a narrower peak to be fit, better matching the filament profiles. The difference was most pronounced for the case of the pure isothermal cylinder model (Equation (5) with $p=4$ ), where few cases produced good fits without a background column density. The pressure-confined model (Equation (7)) almost never converged to a satisfactory fit without a background column density term. The Appendix shows the results of fits with no background column density included.

Figure 6 shows the best fit models for one example filament at $0.1 \mathrm{Myr}$. Following the general behavior seen in the simulations, the filament in the MHD simulation is "fluffier" (wider and lower peak column density) than in the HD simulation. This is a consequence of the significant amount of magnetic pressure support of the filaments as noted earlier.

\subsection{Modified Isothermal Cylinder Model Fits}

As can be seen in Figure 6, the isothermal and modified isothermal cylinder model provide near-identical fits, when a background column density term is included. In both the HD and MHD profiles shown, the models have peak values within the errors of the simulated radial profile. As shown in the Appendix, the models differ by a greater amount when the background column density is fixed to zero, with the pure isothermal model then generally providing a very poor fit.

Table 4 gives the median model fit parameters for filaments where a fit was possible at every time step in both the HD and MHD simulations, to allow for any trends in the time evolution to be followed. The typical powerlaw slopes found $(p \sim 1.3-2)$ are similar to the best-fit values in Arzoumanian et al. (2011). The typical dispersion (standard deviation) in the model fit parameters is of the same size as the median values given in the table; in all cases, there is no clear trend in the best fit parameters evolving as a function of time. Juvela et al. (2012a) and Smith et al. (2014) point out that the modified isothermal fit is partially degenerate between the fit parameters, 
Table 4

Best Fit Parameters for Modified Isothermal Cylinder, with Background

\begin{tabular}{|c|c|c|c|c|c|c|c|c|c|}
\hline \multirow{2}{*}{$\begin{array}{l}\text { Mass } \\
\left(M_{\odot}\right) \\
\end{array}$} & \multirow{2}{*}{$\begin{array}{l}\text { Time } \\
(\mathrm{Myr})\end{array}$} & \multicolumn{3}{|c|}{$\begin{array}{l}\mathrm{HD}^{\mathrm{a}} \\
\end{array}$} & \multicolumn{3}{|c|}{$\mathrm{MHD}^{\mathrm{a}}$} & \multirow[b]{2}{*}{$p$} & \multirow[b]{2}{*}{$\Sigma_{0}$} \\
\hline & & $\rho_{c}$ & $R_{\mathrm{fl}}$ & $p$ & $\Sigma_{0}$ & $\rho_{c}$ & $R_{\mathrm{fl}}$ & & \\
\hline 500 & 0.05 & 0.9 & 3.0 & 2.3 & 5.4 & 1.4 & 3.0 & 2.4 & 7.7 \\
\hline 500 & 0.10 & 14.3 & 0.6 & 1.5 & 3.2 & 5.5 & 2.0 & 1.8 & 3.5 \\
\hline 500 & 0.15 & 8.8 & 0.8 & 1.8 & 8.7 & 1.7 & 3.6 & 3.1 & 4.5 \\
\hline 500 & 0.20 & 4.5 & 2.3 & 4.2 & 9.7 & N/A & N/A & N/A & N/A \\
\hline 2000 & 0.05 & 8.4 & 1.0 & 1.8 & 29 & 6.7 & 1.1 & 1.4 & 23 \\
\hline 500 & $\mathrm{all}^{\mathrm{b}}$ & 4.6 & 1.7 & 2.0 & 4.6 & 1.7 & 2.7 & 2.0 & 4.3 \\
\hline 2000 & $\mathrm{all}^{\mathrm{b}}$ & 8.4 & 1.0 & 1.8 & 29 & 6.7 & 1.2 & 1.4 & 23 \\
\hline
\end{tabular}

Notes.

${ }^{a}$ Mean of the best fit values for filaments fit at all times, with a non-zero background allowed for the fit: the central density, $\rho_{c}$ (in units of $10^{5} \mathrm{~cm}^{-3}$ ), the central flat radius, $R_{\mathrm{fl}}$ (in units of $0.01 \mathrm{pc}$ ), the exponent, $p$, and $\Sigma_{0}$, the background column density term (in units of $10^{21} \mathrm{~cm}^{-2}$ ); the standard deviation is often comparable in magnitude to the mean, with values of $0.6-18,0.4-2.4$, and $0.2-3.5$ for $\rho_{c}, R_{\mathrm{fl}}$ and $p$ respectively, in the same units. The background column density tends to have standard deviations larger than the mean value, and the mean is usually significantly larger than the median.

${ }^{b}$ Values of all profiles where a fit was possible are included here, i.e., relaxing the requirement of a fit for both $\mathrm{HD}$ and $\mathrm{MHD}$, and for the $500 M_{\odot}$ simulations, a fit at all time steps.

which could hide evolutionary trends. Smith et al. (2014) demonstrated that while no time evolution was seen in their best-fit parameters when all were free to vary, fixing $R_{\mathrm{fl}}$ gave best-fit central densities that clearly increased with time. We performed the same test and found a similar result, as shown in Table 5, although the combination of expanding and contracting filaments at later time steps diminishes the strength of the evolutionary trends.

Although the variation between filaments in a single simulation snapshot is larger than any general change in filament behavior as a function of time or initial conditions, the model fits are consistent with the general behaviors noted earlier. Magnetic fields produce puffier (lower $\rho_{c}$ and higher $R_{\mathrm{fl}}$ ) filaments, and an initially higher density tends to lead to more peaky filaments (higher $\rho_{c}$ and lower $R_{\mathrm{ff}}$ ); all of these differences are smaller than the scatter in best fit values for a given simulation snapshot, and would require tracking over a longer time period to better measure time evolution.

As Arzoumanian et al. (2011) found, we also find that the isothermal cylinder model tends to provide a worse fit to the radial column density profile than the modified isothermal model, although this is much more noticeable in the case of a zero background column density (see the Appendix). Typically, the best fit power law index, $p$, is between 1.3 and 2.0, within the range found by Arzoumanian et al. (2011) and others, and much less than $p=4$ for the pure isothermal model. We also find $\rho_{c}$ is around $10^{4}-10^{7} \mathrm{~cm}^{-3}$, and $R_{\mathrm{fl}}$ ranges between roughly 0.001 to $0.1 \mathrm{pc}$, consistent with Juvela et al. (2012b). The relationship between $R_{\mathrm{ff}}$ and the FWHM is dependent on the power law of the profile, and a smaller value of $R_{\mathrm{fl}}$ is expected based on the values of $p$ fitted. Explicitly, $\mathrm{FWHM}=2 \times \sqrt{2^{2 /(p-1)}-1} R_{\mathrm{fl}}$, or $3.46 R_{\mathrm{fl}}$ when $p=2$.

\subsection{Pressure-confined Isothermal Cylinder Model Fits}

The pressure-confined isothermal cylinder model also generally provides a good fit to the filament profiles, although
Table 5

Best Fit Parameters for Modified Isothermal Cylinder, with Background, $R_{\mathrm{fl}}$ Fixed at $0.01 \mathrm{pc}$

\begin{tabular}{|c|c|c|c|c|c|c|c|}
\hline \multirow{2}{*}{$\begin{array}{l}\text { Mass } \\
\left(M_{\odot}\right)\end{array}$} & \multirow{2}{*}{$\begin{array}{l}\text { Time } \\
\text { (Myr) }\end{array}$} & \multicolumn{3}{|c|}{ 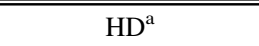 } & \multicolumn{3}{|c|}{$\mathrm{MHD}^{\mathrm{a}}$} \\
\hline & & $\rho_{c}$ & $p$ & $\Sigma_{0}$ & $\rho_{c}$ & $p$ & $\Sigma_{0}$ \\
\hline 500 & 0.05 & 1.6 & 1.6 & 3.2 & 1.6 & 1.4 & 2.5 \\
\hline 500 & 0.10 & 2.7 & 1.8 & 7.0 & 1.9 & 1.5 & 2.6 \\
\hline 500 & 0.15 & 3.3 & 2.1 & 8.6 & 2.2 & 1.6 & 4.2 \\
\hline 500 & 0.20 & 3.6 & 2.0 & 7.3 & N/A & N/A & N/A \\
\hline 2000 & 0.05 & 6.4 & 1.9 & 56 & 6.1 & 2.5 & 55 \\
\hline 500 & all $^{\mathrm{b}}$ & 1.6 & 1.6 & 6.7 & 1.6 & 1.5 & 2.8 \\
\hline 2000 & $\mathrm{all}^{\mathrm{b}}$ & 7.3 & 2.3 & 68 & 8.8 & 2.1 & 58 \\
\hline
\end{tabular}

Notes.

${ }^{a}$ Mean of the best fit values for filaments fit at all times, with a non-zero background allowed for the fit and the central flat radius, $R_{\mathrm{fl}}$ fixed at $0.01 \mathrm{pc}$ : the central density, $\rho_{c}$ (in units of $10^{5} \mathrm{~cm}^{-3}$ ), the exponent, $p$, and $\Sigma_{0}$, the background column density term (in units of $10^{21} \mathrm{~cm}^{-2}$ ); the standard deviation is usually slightly less than the mean, with values of $0.9-2.3$, and $0.2-0.7$ for $\rho_{c}$ and $p$ respectively, in the same units. The background column density tends to have higher standard deviations than the mean values, and the mean is often much higher than the median value.

${ }^{\mathrm{b}}$ Values of all profiles where a fit was possible are included here, i.e., relaxing the requirement of a fit for both $\mathrm{HD}$ and $\mathrm{MHD}$, and for the $500 M_{\odot}$ simulations, a fit at all time steps.

the HD example shown in Figure 6 does a poor job of capturing the peak column density. In most cases, the best-fit model is very similar to the best-fit modified isothermal cylinder model. The mean and standard deviation temperature of all fits are 15 $\pm 14 \mathrm{~K}$, with a small tail in the temperature distribution extending out to $100 \mathrm{~K}$; only $20 \%$ of the temperatures fit were above $20 \mathrm{~K}$; the median temperature fit is $11 \mathrm{~K}$. The typical external pressure fit was $P_{\text {ext }} / k_{B}=4 \pm 3 \times 10^{5} \mathrm{~cm}^{3} \mathrm{~K}^{-1}$, with the tail in the distribution extending up to $10^{6} \mathrm{~cm}^{3} \mathrm{~K}^{-1}$. The typical shape parameters fit were $f=0.76 \pm 0.18$ (keeping in mind $f$ must be between 0 and 1). The fitted temperature and external pressure values are physically reasonable-the temperatures are generally similar to the simulation's constant $10 \mathrm{~K}$, and the typical external pressure is is the same range as those fitted and estimated to be reasonable in nearby molecular clouds such as Perseus (Kirk et al. 2006).

We searched for evolutionary trends within the fitted model parameters, but did not find any over the time period analyzed. The only discernible trend was that the external pressures fit tended to be higher in the $2000 M_{\odot}$ simulations than the $500 M_{\odot}$ simulations, with typical values of $6 \pm 6^{5} \mathrm{~cm}^{3} \mathrm{~K}^{-1}$ and $1 \pm 2 \times 10^{5} \mathrm{~cm}^{3} \mathrm{~K}^{-1}$ respectively. Since the initial density of the $2000 M_{\odot}$ simulation was higher, the external pressure caused by the weight of overlying material within the region would be expected to be higher.

Finally, we made a general comparison of the goodness-offit of the various models, by comparing the typical (mean and standard deviation) chi-squared values of all fits. The standard deviation in chi-squared values is several times larger than the mean for any of the model fits, making a distinction in the overall goodness-of-fit between the models tenuous. Nevertheless, including a background column density term for the isothermal cylinder model made a substantial difference: the mean chi-squared value for fits with a background included is 


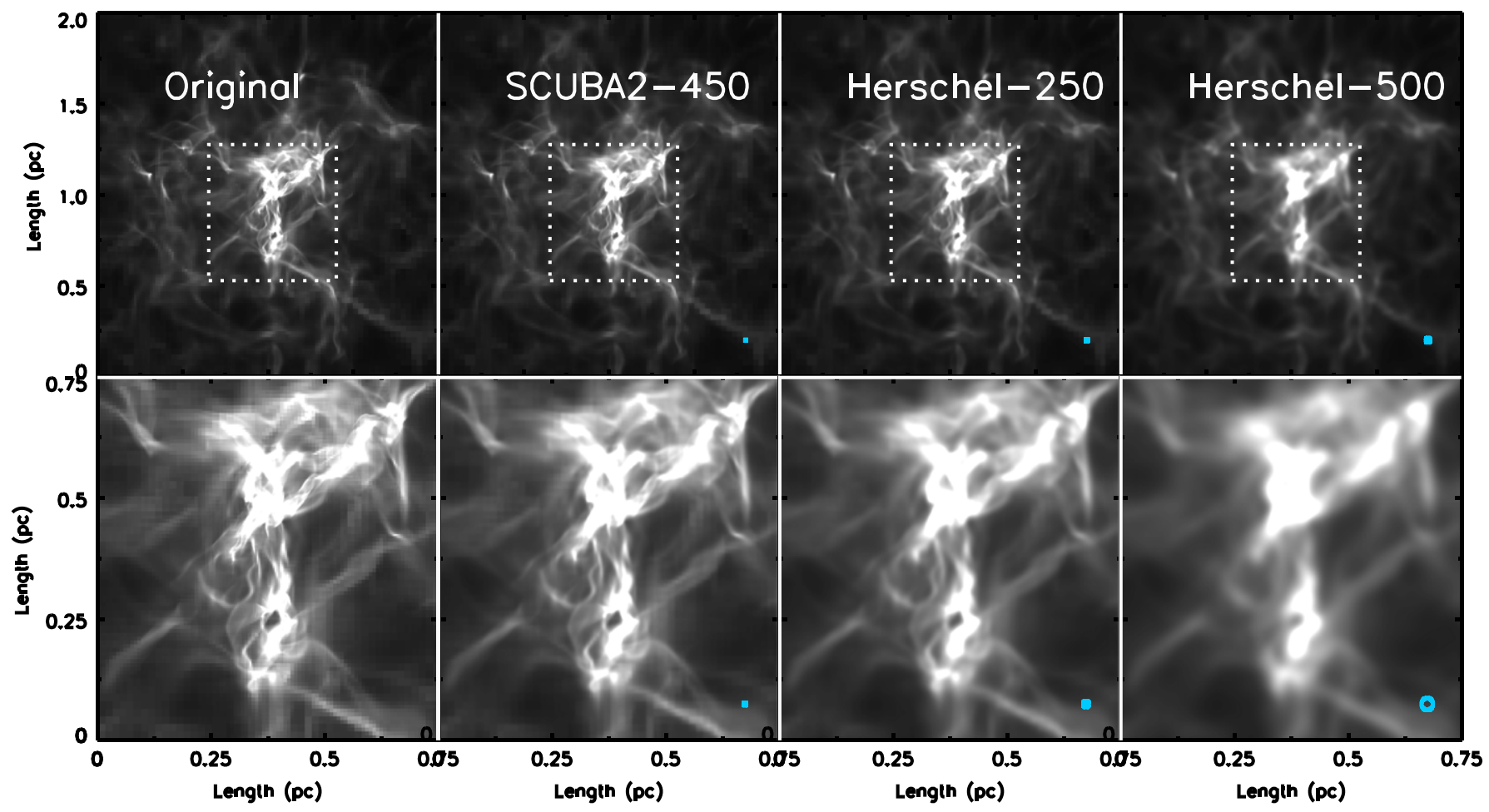

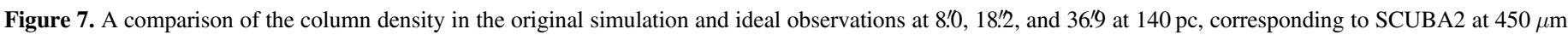

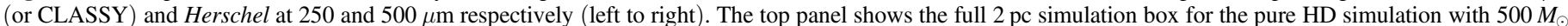


for each panel.

more than 3.5 times smaller than when the background is zero. The difference is much less pronounced for the modified isothermal model where including a background column density decreases the mean chi-squared value by $40 \%$. Allowing the power law to vary (purely isothermal model versus modified isothermal model) yields a $30 \%$ improvement in the mean chi-squared value, while the pressure-confined model has a mean chi-squared value $8 \%$ lower than the modified isothermal model.

\section{EFFECT OF RESOLUTION}

Finally, we examine the effect of resolution on our results. Real observations of filamentary structures are complicated by both instrumental effects (including resolution and system noise) and physical effects (how the flux emitted at a specific wavelength relates to the intrinsic column density of material), which make direct comparisons between simulations and observations difficult (e.g., Goodman et al. 2009). Here, we consider only the simplest factor, spatial resolution, to represent what "perfect" observations would be able to reveal. For the analysis presented here, we assume that the observed system is located $140 \mathrm{pc}$ away, representing the very nearest molecular clouds and therefore also a best-case scenario. While we tested a variety of resolutions, we show results from three cases which are representative of the present single-dish facilities able to map large areas of the sky in a reasonable amount of time. The first case we consider is a resolution of $8^{\prime \prime}$. This corresponds to the resolution of the James Clerk Maxwell telescope (JCMT) at $450 \mu \mathrm{m}$ (Holland et al. 2013), and is also similar to the $7^{\prime \prime}$ resolution of the recent CLASSY survey (e.g., FernándezLópez et al. 2014) which studies in detail three nearby molecular clouds in unprecedented detail with the CARMA interferometer. The second case we consider is a resolution of 18'.2 corresponding to the resolution of Herschel column density maps using the group's latest standard method (e.g., Palmeirim et al. 2013), with the resolution corresponding to that of their $250 \mu \mathrm{m}$ observations. The third and final case we consider is a resolution of 36.9 corresponding to the resolution of Herschel at $500 \mu \mathrm{m}$, and earlier Herschel column density maps (e.g., Könyves et al. 2010; Arzoumanian et al. 2011).

Figure 7 shows an example of the effect of a resolution on the column density in the simulation. As is clearly illustrated by this comparison, at the longest wavelength Herschel resolutions, much of the fine filamentary structure is lost, while SCUBA2 at $450 \mu \mathrm{m} / \mathrm{CLASSY}$ does a better job.

We also examined the quantitative effect of the resolution on our results. To do this, we applied the same filament definitions and re-ran our analysis. We attempted to correct for the resolution in a similar manner to real observational analyses: for the direct filament FWHM measurements, we deconvolved the values with the resolution. For the column density profile models, we convolved the model with the "beam profile"/ resolution before fitting. We find that despite attempting to correct for the resolution during analysis using the standard observational techniques, the poorest resolution still gives biased results. A full comparison of the effect of resolution on all of our measured quantities is given in Table 6: we calculated the mean and standard deviation of the ratio in values between the decreased resolution and original results. In summary, while there is a significant amount of scatter, typically the resolution only produces a somewhat noticeable effect for the 36.9 or Herschel $500 \mu \mathrm{m}$ case. The effects tend to be in the direction expected: poorer resolution leads to higher widths and 
Table 6

Effect of Resolution on Fit Parameters ${ }^{\mathrm{a}}$

\begin{tabular}{lccc}
\hline \hline Resolution & FWHM $^{b}$ & $\sigma^{b}$ & $M_{\text {line }}^{b}$ \\
\hline JCMT-450 $\mu \mathrm{m}$ & $1.2 \pm 0.4$ & $1.1 \pm 0.2$ & $1.1 \pm 0.2$ \\
Herschel $-250 \mu \mathrm{m}$ & $1.3 \pm 0.5$ & $1.1 \pm 0.2$ & $1.2 \pm 0.3$ \\
Herschel-500 $\mu \mathrm{m}$ & $1.6 \pm 1.2$ & $1.3 \pm 0.4$ & $1.4 \pm 0.6$ \\
\hline Resolution & $\rho_{c}^{c}$ & $R_{\mathrm{fl}}^{c}$ & $p^{c}$ \\
\hline JCMT-450 $\mu \mathrm{m}$ & $0.8 \pm 0.3$ & $1.8 \pm 1.3$ & $1.2 \pm 0.4$ \\
Herschel $-250 \mu \mathrm{m}$ & $1.1 \pm 1.1$ & $1.5 \pm 1.1$ & $1.1 \pm 0.5$ \\
Herschel-500 $\mu \mathrm{m}$ & $0.5 \pm 0.3$ & $3.4 \pm 2.6$ & $1.6 \pm 0.7$ \\
\hline Resolution & $\rho_{c}^{d}$ & $R_{\mathrm{fl}}^{d}$ & $p^{d}$ \\
\hline JCMT-450 $\mu \mathrm{m}$ & $1.1 \pm 0.6$ & $1.1 \pm 0.3$ & $\ldots$ \\
Herschel- $250 \mu \mathrm{m}$ & $1.1 \pm 0.6$ & $1.1 \pm 0.3$ & $\ldots$ \\
Herschel-500 $\mu \mathrm{m}$ & $0.8 \pm 0.4$ & $1.5 \pm 0.7$ & $\ldots$ \\
\hline Resolution & $T^{e}$ & $P_{\mathrm{ext}}^{e}$ & $f_{c y l}^{e}$ \\
\hline JCMT-450 $\mu \mathrm{m}$ & $1.1 \pm 0.2$ & $1.2 \pm 0.5$ & $1.0 \pm 0.2$ \\
Herschel-250 $\mu \mathrm{m}$ & $1.2 \pm 0.6$ & $1.3 \pm 0.6$ & $1.0 \pm 0.2$ \\
Herschel-500 $\mu \mathrm{m}$ & $1.4 \pm 0.7$ & $1.4 \pm 0.8$ & $1.0 \pm 0.3$ \\
\hline
\end{tabular}

Notes.

a Mean and standard deviation in the ratio between decreased resolution fits and original values (all quantities).

${ }^{\mathrm{b}}$ Filament FWHM widths, Gaussian-fitted widths, and mass per unit length ratio.

${ }^{c}$ Parameters from the modified isothermal cylinder model.

d Parameters from the isothermal cylinder model (power law exponent fixed).

e Parameters from the pressure-confined isothermal cylinder model.

lower central densities. We emphasize that these tests correspond to the best possible case for these instruments. Very few star-forming clouds are as close as $140 \mathrm{pc}$; even Perseus is nearly twice as far away at $\sim 250 \mathrm{pc}$, and many other Gould Belt Survey clouds are at a similar or larger distance. Filaments surveyed in the Herschel Hi-Gal Survey are several times more distant still. We ran additional tests (not shown) with even poorer spatial resolution, corresponding to filaments at these further distances, and found that the biases in observed quantities becomes much more noticeable in those cases.

Smith et al. (2014) tested the effect of resolution on measured filament widths and find relatively little effect. Their test used a degraded resolution of 0.0086 pc (R. Smith 2015, private communication), corresponding to a resolution of $\sim 13^{\prime \prime}$ at $140 \mathrm{pc}$. Juvela et al. (2012b) also examined the effect of resolution on simulated filaments, using an angular resolution of $40^{\prime \prime}$ at 93, 186, and $371 \mathrm{pc}$. They found that at the larger distances, the filament central densities were the most biased, while the width, mass per unit length, and power law slope changed by less. Both of these results are consistent with our findings at similar resolutions.

Beyond the implications to the measured filament properties presented here is the presence and characterization of substructure within the filaments. Averaging a radial column density profile along a filament hides much of the information on smaller-scale structure within the filaments in the simulations - see, for example, the comparison of 2D and 3D column density versus density profiles of simulated filaments in Gómez \& Vázquez-Semadeni (2014) and Smith et al. (2014). Both observations (Hacar et al. 2013; Fernández-López et al. 2014; Henshaw et al. 2014) and simulations (Moeckel \& Burkert 2014; Smith et al. 2014) suggest that filaments may in fact be composed of multiple strands of dense gas, perhaps woven together, which are often difficult to identify separately with the present observational capabilities; higher spatial resolution and/ or inclusion of the line of sight velocity are essential.

In our hydrodynamic simulations too (see Figure 7), we see evidence of more complex structure on smaller scales, although a full 3D consideration of this is beyond the scope of the present paper. Nonetheless, our results coupled with new results emerging from observations such as Fernández-López et al. (2014) suggest that the key to a deeper understanding of filamentary structure requires high (spatial and velocity) resolution as well as more sophisticated tools by which to model the structure.

\section{DISCUSSION AND CONCLUSIONS}

We simulate the formation of filaments within a clump-scale volume, investigating the role of magnetic fields on the evolution of filaments column density. Starting with $500 M_{\odot}$ (or $2000 M_{\odot}$ ) of gas within a 2 pc cube, we track the evolution of filamentary structures over $0.15 \mathrm{Myr}$ with and without the presence of a magnetic field. Turbulence remains strong throughout the simulations as there has been been insufficient time for it to damp significantly.

These analyses provide an important complement to the recent filamentary analysis in simulations by Smith et al. (2014). Those authors investigated the effect of different initial modes of turbulence (e.g., solenoidal versus compressive), whereas our analysis examines the effect of magnetic fields and gravity (through varying the initial mean density). Other more subtle differences are also important to note as well. Smith et al. (2014) assumed a uniform density initial sphere, surrounded by a warm diffuse medium, whereas we assume a sphere with a radially decreasing density surrounded by a vacuum. Girichidis et al. (2011) demonstrate that the initial density distribution can have a marked effect on the large-scale structure which forms later in the simulation. Our simulations do not include the effect of radiation or simple chemistry, while Smith et al. (2014) does include both; we expect these effects to become more important at later times (once massive YSOs begin ionizing their natal environments) and when making synthetic observations of molecular line emission (where the presence or absence of various molecular species has a large effect). The base numerical codes used are also different-Smith et al. (2014) use AREPO, a hybrid code, while we use FLASH, which is an AMR-based code. Despite these significant differences, both Smith et al. (2014) and our analyses do identify filaments that have properties broadly similar to observed filaments, which appears to speak to the universality of filament formation under a variety of conditions.

We also note one major difference from Smith et al. (2014) in the analysis stage: Smith et al. (2014) focus their analysis on the brightest one or two filaments in each simulation whereas we also include fainter/less dense filaments in our analysis. In this respect, our analysis gives a better direct comparison with observational surveys, where many filaments are identified in any given star-forming region. Our approach limits our analysis of the filament column density profiles to a smaller radial separation from the filament spine, since the fainter filaments are more liable to have their profiles contaminated by nearby 
non-related substructure than brighter filaments are (the filling factor is much larger for all faint plus bright filaments than it is for just bright filaments). On the other hand, inclusion of fainter filaments allows us greater sensitivity to less gravitationally bound filaments, which may be more liable to re-expand with time; such behavior was not noted in Smith et al. (2014), presumably because the dominant filament in each simulation will continue to contract and accrete new material throughout time. Our main findings are as follows:

1. Magnetic fields have a strong influence on filamentary structure. Even with a mass to magnetic flux ratio which is supercritical by a factor of $\sim 2$, there are notable differences from the purely hydrodynamic simulation. Filaments formed in the magnetic case tend to be wider, less centrally peaked, and evolve more slowly than filaments seen in the purely hydrodynamic case. These differences are most apparent through a visual comparison (e.g., Figures 2-4), and are less discernible in quantitative measures due to the large variation in filament properties at any given snapshot.

2. The magnetic field can have a strong effect on the fragmentation of filaments. In our simulations, magnetic fields are able to significantly suppress the formation of cores, since its energy density exceeds that of the turbulence. The turbulence is sub-Alfvénic $\left(\mathcal{M}_{A}=v_{A} / \sigma \sim 2.1-2.2\right)$ and so the magnetic field increases the critical turbulent line mass by a factor of 3.2-3.3. This accounts for the less condensed structure of the magnetized filaments, and their notably less fragmentation.

3. The simulated filaments have properties consistent with observations. The radial column density profiles of the filaments are well-described by a Plummer-like or modified isothermal cylinder profile. The power-law slope tends to be around 1.3-2, similar to the range of 1.5-2.5 found in Herschel data byArzoumanian et al. (2011). The central density tends to be of order $10^{5} \mathrm{~cm}^{-3}$ for the $500 M_{\odot}$ simulations and closer to $10^{6} \mathrm{~cm}^{-3}$ for the $2000 M_{\odot}$ simulations; the inner flat radius is a few hundredths of a parsec in both cases. The $500 M_{\odot}$ typical central densities, as well as the inner flat radii and power law slopes, are consistent with those in Juvela et al. (2012a), also based on Herschel observations. The pressure-confined cylinder model of Fischera \& Martin (2012) also provides a reasonable fit to the radial column density profiles, with typical temperatures, external pressures, and shape parameters fit of $15 \mathrm{~K}, 4 \times 10^{5}$ $\mathrm{cm}^{3} \mathrm{~K}^{-1}$, and 0.76 for $T, P_{\mathrm{ext}} / k_{B}$, and $f$ respectively.

4. Filaments have diverse evolutionary paths. At any given snapshot in time, the simulation reveals a variety of filaments. Some continue to radially contract and accrete material throughout the simulation and will presumably continue on to form stars along their length. Other filaments halt in their contraction and expand into the ambient medium before the end of the simulation. Given the relatively short time duration of our simulations, we expect that even more diverse evolutionary paths could be possible throughout the lifetime of a molecular cloud.

5. The mass per unit length of a filament in a given snapshot provides only a weak discriminant between the contracting and expanding filaments. Over most of the range of mass per unit length values, filaments can be either contracting or expanding. Above roughly $72 M_{\odot} \mathrm{pc}^{-1}$, the critical value for filaments supported equally by thermal and non-thermal support, nearly all filaments are contracting. Velocity and magnetic field information are clearly required to determine the evolutionary state of a filament unambiguously.

6. Turbulence plays an important role in the mass per unit length of filaments. The filaments in which stars appear in our simulations have critical mass per unit lengths that are dominated by turbulent velocity dispersion and not just thermal values. This arises in these simulations since they are much less than a free-fall time old, so that turbulence has not had the opportunity to damp significantly. Herschel results indicate that the thermal critical value of $M_{\text {line }}^{\text {crit }}$ is a good diagnostic of star-forming ability therefore suggests that turbulence is much weaker within these filaments, perhaps because their natal clouds are more evolved.

7. Filament widths are mildly influenced by environment. Filaments tend to be narrower at later times, when formed in higher density environments, or without the presence of magnetic fields, but these trends are all weak, given the mixture of contracting and expanding filaments at every snapshot in the simulations. Stronger trends in the evolution in filament properties as a function of time might become apparent with a longer timescale for analysis, especially if the filaments which re-expand become sufficiently diffuse to become undetectable at later times. We find the mean filament FWHM ranges from 0.06 to $0.26 \mathrm{pc}$ across our simulations at varying times, with most being around $0.1-0.15 \mathrm{pc} .{ }^{10}$ The range in filament FWHM values for any given simulation snapshot is, however, larger than the range seen in the analyses of Arzoumanian et al. (2011). For filaments which are contracting, a combination of the decaying turbulence and gravity is likely responsible for the evolution. This may in part explain the relative constant filament widths discussed in Arzoumanian et al. (2011), although resolution may also be an important factor (Fernández-López et al. 2014), and observational biases and measurement methods could be playing a role (Heitsch 2013a; Smith et al. 2014).

8. Filaments have complex structures. The radial column density profiles of the filaments reveal a wealth of substructure, particularly in the pure hydrodynamic simulation where features tend to be sharper. Some of these substructures appear suggestively like the intertwined filament bundles found by Hacar et al. (2013; see, for example, the bottom left panel in Figure 7); highresolution observations of a suite of filaments will be necessary to show how commonplace this phenomenon is. Other simulations, including Smith et al. (2014) and Moeckel \& Burkert (2014) also find complex 3D filamentary substructure.

Future work will include a 3D analysis of the filaments formed in these simulations, including their velocity structure and accretion rates, and the relationship with the magnetic field geometry.

\footnotetext{
${ }^{10}$ See Section 3.1 for a discussion on the biases and uncertainties in absolute filament widths in our analyses.
} 


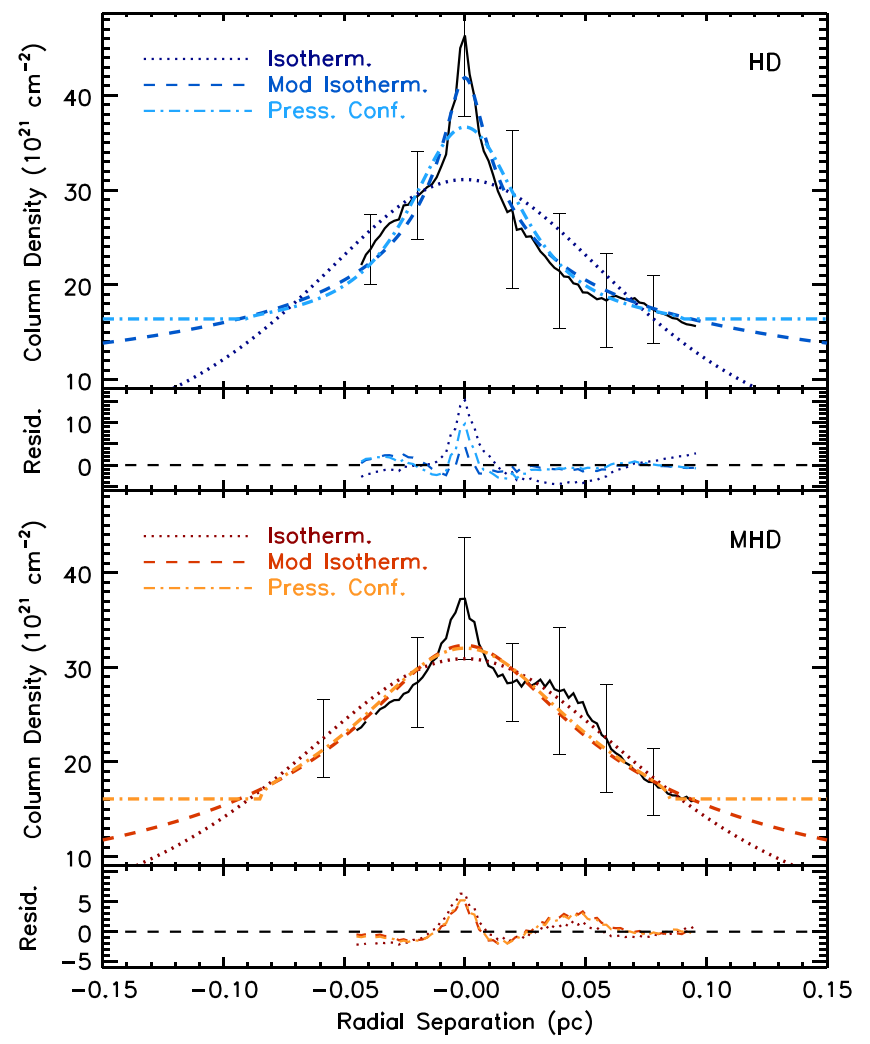

Figure 8. The radial column density profile and best fit models for a filament, including the fitting residuals where the background column density level for the isothermal and modified isothermal cylinder models is fixed to zero. See Figure 6 for the plotting conventions used. Note that the vertical range in the residual plots differs between the upper and lower profiles. In this case, the pure isothermal cylinder model is not a good match to the profile.

We thank the referee for a thoughtful and thorough review which helped to strengthen our paper. M.K. thanks Philip Girichidis for sharing his turbulence generator with us, and Thierry Sousbie for providing us with a pre-release version of DisPerSE. H.K. thanks Rowan Smith for helpful discussions about analyzing filaments in numerical simulations, especially in comparing her recent results with ours. H.K. thanks Doris Arzoumanian for providing some clarification to the radial column density profile fitting method used by the Herschel team. H.K. also thanks Doug Johnstone and Phil Myers for useful discussions about various aspects of this work. H.K. acknowledges support from the Banting Postdoctoral Fellowships program, administered by the Government of Canada. M.K. acknowledges financial support from the National Sciences and Engineering Research Council (NSERC) of Canada through a Postgraduate Scholarship. R.E.P. is supported by a Discovery Grant from the Natural Sciences and Engineering Research Council (NSERC) of Canada. The FLASH code was in part developed by the DOE NNSA-ASC OASCR Flash Center at the University of Chicago. This work was made possible by the facilities of the Shared Hierarchical Academic Research Computing Network (SHARCNET: http://www. sharcnet.ca and Compute/Calcul Canada.

\section{APPENDIX}

Here, we examine the results of fits to the radial column density profiles of the filaments when the background column density is forced to be zero (see discussion in Section 4).
Table 7

Best Fit Parameters for Modified Isothermal Cylinder, No Background

\begin{tabular}{|c|c|c|c|c|c|c|c|}
\hline \multirow{2}{*}{$\begin{array}{l}\text { Mass } \\
\left(M_{\odot}\right)\end{array}$} & \multirow{2}{*}{$\begin{array}{l}\text { Time } \\
(\mathrm{Myr})\end{array}$} & \multicolumn{3}{|c|}{$\mathrm{HD}^{\mathrm{a}}$} & \multicolumn{3}{|c|}{$\overline{\text { MHD }^{\mathrm{b}}}$} \\
\hline & & $\rho_{c}$ & $R_{\mathrm{fl}}$ & $p$ & $\rho_{c}$ & $R_{\mathrm{ff}}$ & $p$ \\
\hline$\overline{500}$ & 0.05 & 1.1 & 1.7 & 1.4 & 0.7 & 2.0 & 1.4 \\
\hline 500 & 0.10 & 3.1 & 0.7 & 1.4 & 0.7 & 1.8 & 1.4 \\
\hline 500 & 0.15 & 3.9 & 0.6 & 1.5 & 2.3 & 1.2 & 1.4 \\
\hline 500 & 0.20 & 2.6 & 0.8 & 1.5 & N/A & N/A & N/A \\
\hline 2000 & 0.05 & 11.1 & 0.7 & 1.3 & 7.9 & 0.6 & 1.2 \\
\hline 500 & $\mathrm{all}^{\mathrm{b}}$ & 2.4 & 0.7 & 1.4 & 1.0 & 1.6 & 1.4 \\
\hline 2000 & $\mathrm{all}^{\mathrm{b}}$ & 11.1 & 0.5 & 1.2 & 10.7 & 0.6 & 1.3 \\
\hline
\end{tabular}

Notes.

${ }^{a}$ Median of the best fit values for filaments fit at all times: the central density, $\rho_{c}$ (in units of $10^{5} \mathrm{~cm}^{-3}$ ), the central flat radius, $R_{\mathrm{fl}}$ (in units of $0.01 \mathrm{pc}$ ), and the exponent, $p$; the standard deviation is often comparable in magnitude to the median, with values of $0.7-32,0.7-4.4$, and $0.2-0.5$ respectively, in the same units.

${ }^{\mathrm{b}}$ Values of all profiles where a fit was possible are included here, i.e., relaxing the requirement of a fit for both $\mathrm{HD}$ and $\mathrm{MHD}$, and for the $500 M_{\odot}$ simulations, a fit at all time steps.

Figure 8 shows the best fit models for the isothermal and modified isothermal model, for a filament identified in both the HD (top panel) and MHD (bottom panel) $500 M_{\odot}$ simulations, to be contrasted with Figure 6 for the same fits where background column density is allowed to be non-zero. Note that the best-fit pressure-confined model plotted in Figure 8 does include a background column density term, and is identical to the fit shown in Figure 6. Contrary to the case (Section 4) when the background column density is fixed to zero, the three models differ from each other by a greater amount, and the pure isothermal model is generally a poor fit to the profile.

Table 7 gives the median model fit parameters for the isothermal and modified isothermal profiles for all filaments fitted at every time step in both the HD and MHD simulations. Comparison of these fit values with those given in Table 4 shows that the best fit profiles tend to have narrower peaks (better matching the filament profiles) when a background column density included, most of this increased narrowness is accounted for by the steeper power law, $p$, rather than a decrease in the central flat radius, $R_{\mathrm{fl}}$. As discussed in Section 4.2 , there is relatively little difference in the quality of fit (comparing typical $\chi^{2}$ values) for the modified isothermal model when a background column density is or is not included, however, the inclusion of a background column density terms makes a substantial difference in the quality of fits for the purely isothermal model.

\section{REFERENCES}

Alves, J., Lada, C. J., Lada, E. A., Kenyon, S. J., \& Phelps, R. 1998, ApJ, 506, 292

André, P., Men'shchikov, A., Bontemps, S., et al. 2010, A\&A, 518, L102

Arzoumanian, D., André, P., Didelon, P., et al. 2011, A\&A, 529, L6

Arzoumanian, D., André, P., Peretto, N., \& Könyves, V. 2013, A\&A, 553, A119

Bally, J., Lanber, W. D., Stark, A. A., \& Wilson, R. W. 1987, ApJL, 312, L45 Banerjee, R., Pudritz, R. E., \& Anderson, D. W. 2006, MNRAS, 373, 1091

Banerjee, R., Vázquez-Semadeni, E., Hennebelle, P., \& Klessen, R. S. 2009, MNRAS, 398, 1082

Bergin, E. A., \& Tafalla, M. 2007, ARA\&A, 45, 339 
Beuther, H., Vlemmings, W. H. T., Rao, R., \& van der Tak, F. F. S. 2010, ApJL, 724, L113

Boldyrev, S. 2002, ApJ, 569, 841

Bourke, T. L., Myers, P. C., Caselli, P., et al. 2012, ApJ, 745, 117

Contreras, Y., Rathborne, J., \& Garay, G. 2013, MNRAS, 433, 251

Crutcher, R. M., Wandelt, B., Heiles, C., Falgarone, E., \& Troland, T. H. 2010, ApJ, 725, 466

Falgarone, E., Troland, T. H., Crutcher, R. M., \& Paubert, G. 2008, A\&A, 487, 247

Federrath, C., Banerjee, R., Clark, P. C., \& Klessen, R. S. 2010, ApJ, 713, 269

Federrath, C., Klessen, R. S., \& Schmidt, W. 2008, ApJL, 688, L79

Fernández-López, M., Arce, H. G., Looney, L., et al. 2014, ApJL, 790, L19

Fiege, J. D., \& Pudritz, R. E. 2000, MNRAS, 311, 85

Fischera, J., \& Martin, P. G. 2012, A\&A, 542, A77

Foster, J. B., Rosolowsky, E. W., Kauffmann, J., et al. 2009, ApJ, 696, 298

Fryxell, B., Olson, K., Ricker, P., et al. 2000, ApJS, 131, 273

Girart, J. M., Beltrán, M. T., Zhang, Q., Rao, R., \& Estalella, R. 2009, Sci, 324,1408

Girichidis, P., Federrath, C., Banerjee, R., \& Klessen, R. S. 2011, MNRAS, 413, 2741

Gómez, G. C., \& Vázquez-Semadeni, E. 2014, ApJ, 791, 124

Goodman, A. A., Pineda, J. E., \& Schnee, S. L. 2009, ApJ, 692, 91

Hacar, A., \& Tafalla, M. 2011, A\&A, 533, A34

Hacar, A., Tafalla, M., Kauffmann, J., \& Kovács, A. 2013, A\&A, 554, A55

Hatchell, J., Wilson, T., Drabek, E., et al. 2013, MNRAS, 429, L10

Heitsch, F. 2013a, ApJ, 769, 115

Heitsch, F. 2013b, ApJ, 776, 62

Hennebelle, P., \& André, P. 2013, A\&A, 560, A68

Hennemann, M., Motte, F., Schneider, N., et al. 2012, A\&A, 543, L3

Henshaw, J. D., Caselli, P., Fontani, F., Jiménez-Serra, I., \& Tan, J. C. 2014, MNRAS, 440, 2860

Heyer, M. H., \& Brunt, C. M. 2004, ApJL, 615, L45

Hill, T., André, P., Arzoumanian, D., et al. 2012, A\&A, 548, L6

Holland, W. S., Bintley, D., Chapin, E. L., et al. 2013, MNRAS, 430, 2513

Inutsuka, S.-I., \& Miyama, S. M. 1997, ApJ, 480, 681

Johnstone, D., di Francesco, J., \& Kirk, H. 2004, ApJL, 611, L45

Juvela, M., Malinen, J., \& Lunttila, T. 2012a, A\&A, 544, A141

Juvela, M., Ristorcelli, I., Pagani, L., et al. 2012b, A\&A, 541, A12

Kauffmann, J., Pillai, T., Shetty, R., Myers, P. C., \& Goodman, A. A. 2010 , ApJ, 716, 433

Kirk, H., Johnstone, D., \& di Francesco, J. 2006, ApJ, 646, 1009

Kirk, H., Myers, P. C., Bourke, T. L., et al. 2013, ApJ, 766, 115

Kirk, H., Pineda, J. E., Johnstone, D., \& Goodman, A. 2010, ApJ, 723, 457
Klassen, M., Pudritz, R. E., \& Peters, T. 2012, MNRAS, 421, 2861

Klessen, R. S., Heitsch, F., \& mac Low, M.-M. 2000, ApJ, 535, 887

Könyves, V., André, P., Schneider, N., et al. 2013, AN, 334, 908

Könyves, V., André, P., Men'shchikov, A., et al. 2010, A\&A, 518, L106

Lada, C. J., Alves, J., \& Lada, E. A. 1999, ApJ, 512, 250

Larson, R. B. 1981, MNRAS, 194, 809

MacNeice, P., Olson, K. M., Mobarry, C., de Fainchtein, R., \& Packer, C. 2000, CoPhC, 126, 330

Malinen, J., Juvela, M., Rawlings, M. G., et al. 2012, A\&A, 544, A50

Markwardt, C. B. 2009, in ASP Conf. Ser. 411, Astronomical Data Analysis Software and Systems XVIII, ed. D. A. Bohlender, D. Durand, \& P. Dowler (San Francisco, CA: ASP), 251

Moeckel, N., \& Burkert, A. 2014, arXiv

Motte, F., Zavagno, A., Bontemps, S., et al. 2010, A\&A, 518, L77

Mouschovias, T. C., \& Spitzer, L., Jr. 1976, ApJ, 210, 326

Myers, P. C. 2009, ApJ, 700, 1609

Myers, P. C. 2011, ApJ, 735, 82

Nutter, D., Kirk, J. M., Stamatellos, D., \& Ward-Thompson, D. 2008 , MNRAS, 384, 755

Offner, S. S. R., Klein, R. I., McKee, C. F., \& Krumholz, M. R. 2009, ApJ, 703,131

Olson, K. M., MacNeice, P., Fryxell, B., et al. 1999, BAAS, 31, 1430

Ostriker, J. 1964, ApJ, 140, 1056

Palmeirim, P., André, P., Kirk, J., et al. 2013, A\&A, 550, A38

Peretto, N., André, P., Könyves, V., et al. 2012, A\&A, 541, A63

Peretto, N., Fuller, G. A., \& André, P. 2014, A\&A, 561, A83

Pineda, J. E., Goodman, A. A., Arce, H. G., et al. 2011, ApJL, 739, L2

Pirogov, L. E. 2009, ARep, 53, 1127

Plummer, H. C. 1911, MNRAS, 71, 460

Recchi, S., Hacar, A., \& Palestini, A. 2013, A\&A, 558, A27

Recchi, S., Hacar, A., \& Palestini, A. 2014, MNRAS, 444, 1775

Rosolowsky, E. W., Pineda, J. E., Foster, J. B., et al. 2008, ApJS, 175, 509

Schnee, S., Rosolowsky, E., Foster, J., Enoch, M., \& Sargent, A. 2009, ApJ, 691,1754

Schneider, N., Csengeri, T., Hennemann, M., et al. 2012, A\&A, 540, L11

Schneider, S., \& Elmegreen, B. G. 1979, ApJS, 41, 87

Seifried, D., Banerjee, R., Klessen, R. S., Duffin, D., \& Pudritz, R. E. 2011, MNRAS, 417, 1054

Smith, R. J., Glover, S. C. O., \& Klessen, R. S. 2014, MNRAS, 445, 2900

Sousbie, T. 2011, MNRAS, 414, 350

Sousbie, T., Pichon, C., \& Kawahara, H. 2011, MNRAS, 414, 384

Walawender, J., Bally, J., Francesco, J. D., Jørgensen, J., \& Getman, K. 2008, in Handbook of Star-Forming Regions, Vol. I: The Northern Sky, ed B. Reipurth (San Francisco, CA: ASP), 346 